Matthias Mader/Harald Schoen

\title{
Alles eine Frage des Blickwinkels?
}

Framing-Effekte und Bevölkerungsurteile über einen möglichen Bundeswehreinsatz in Libyen

Der Aufsatz beschäftigt sich mit der Frage, ob in Deutschland trotz antimilitaristischer Grundhaltungen öffentliche Unterstützung zu militärischen Auslandseinsätzen durch Kommunikationsstrategien erzeugt werden kann. Am Fall des im Jahr 2011 kurzzeitig diskutierten Bundeswehreinsatzes in Libyen wird mit einem Umfrageexperiment untersucht, ob sich Bewertungen seitens der Bevölkerung verändern, wenn dessen militärischer Charakter oder dessen humanitäre Zielsetzung betont werden. Die Ergebnisse zeigen, dass die öffentliche Zustimmung unverändert bleibt, wenn auf die Gefahr militärischer Auseinandersetzungen verwiesen wird. Wird dagegen die humanitäre Zielsetzung in den Vordergrund gerückt, erhöht sich die Zustimmung substanziell. Diese Verschiebung ist bei jenen Bürgern besonders groß, die eine aktive deutsche Außenpolitik befürworten und sich dem linken politischen Lager zuordnen.

\section{Einleitung}

Seit Ende des Kalten Krieges und der Wiedervereinigung wird die Bundeswehr in zunehmendem Maße in multilateral geführte, militärische Auslandseinsätze geschickt. Sowohl die Anzahl der eingesetzten Soldaten als auch die Intensität der Konflikte, in die sie dabei verwickelt wurde, haben zugenommen und in der Beteiligung am ISAF-Einsatz in Afghanistan wohl ihren vorläufigen Höhepunkt erreicht (Baumann/Hellmann 2001: 66; Gareis 2011: 156-161; Meiers 2010: 217; Risse 2004: 28). Bei Entscheidungen für oder gegen derartige Einsätze berücksichtigen politische Entscheidungsträger auch die öffentliche Meinung, ${ }^{1}$ nicht zuletzt weil sie andernfalls mit einer sinkenden Unterstützung der Bevölkerung in der Wahlkabine rechnen müssen. ${ }^{2}$ Um derartige Reaktionen zu vermeiden, werben Politiker in der Öffentlichkeit für die von ihnen vertretenen Positionen. Während zur Wirksamkeit derartiger Kommunikationsstrategien bei innenpolitischen Sachfragen eine Vielzahl

1 Vgl. Jacobs/Shapiro (1999); Page/Shapiro (1983) und Sobel (2001) für Beiträge aus der außen- und sicherheitspolitischen Einstellungs- und Verhaltensforschung. Zur Bedeutung gesellschaftlicher Faktoren allgemein siehe beispielsweise die Beiträge zu Zwei-Ebenen-Modellierungen Moravcsik (1997); Putnam (1988) und dem Konzept der strategischen Kultur Johnston (1995); Snyder (1977).

2 Vgl. die Beiträge von Aldrich et al. (2006; 1989); Brooks et al. (2010); Norpoth/Sidman (2007); Schoen (2010; 2011) und Weisberg/Christenson (2007). 
an Forschungsergebnissen vorliegt, ${ }^{3}$ ist nichts darüber bekannt, ob sich auch die Bevölkerungsbewertungen militärischer Auslandseinsätze auf diese Weise verschieben lassen.

In besonderem Maße stellt sich diese Frage für die Bundesrepublik. Die deutsche Bevölkerung vertritt mehrheitlich antimilitaristische Grundhaltungen und neigt dazu, konkrete Bundeswehreinsätze nicht vor dem Hintergrund situationsspezifischer Faktoren, sondern der kategorischen Ablehnung des Einsatzes militärischer Mittel zu beurteilen. ${ }^{4} \mathrm{Ob}$ politische Entscheidungsträger öffentliche Unterstützung zu konkreten Bundeswehreinsätzen mithilfe von Kommunikationsstrategien erzeugen können, ist daher besonders fraglich. Der vorliegende Aufsatz liefert erste Befunde über die Wirkung solcher Kommunikationsstrategien am Beispiel eines möglichen Einsatzes der Bundeswehr in Libyen, der in Deutschland im Frühjahr 2011 vor dem Hintergrund des Aufstandes gegen den autoritär regierenden Machthaber Muammar al-Gaddafi kurzzeitig diskutiert wurde.

Der Libyen-Fall bietet sich in besonderem Maße für eine derartige Analyse an, da es sich hierbei eindeutig um einen Kampfeinsatz handelte. Dies wurde in der innenpolitischen Debatte über die mögliche Beteiligung der Bundeswehr von der Bundesregierung explizit betont (Merkel 2011: 11251; Wittig 2011: 5) und dürfte daher einem Großteil der Bevölkerung bekannt gewesen sein. Aufgrund der kritischen Haltung der Deutschen zu dem Einsatz militärischer Mittel standen die Erfolgschancen von Kommunikationsstrategien besonders schlecht, Unterstützung für einen Einsatz zu gewinnen, bei dem es mit an Sicherheit grenzender Wahrscheinlichkeit zu militärischen Auseinandersetzungen kommen würde. Es handelt sich demnach um einen vergleichsweise harten Test der Wirksamkeit einer Kommunikationsstrategie, die Unterstützung für einen Bundeswehreinsatz schaffen soll.

Die Forschungsfrage wird mithilfe eines Umfrageexperiments untersucht. Es werden (multivariate) lineare Regressionsmodelle gerechnet, in denen die Unterstützung eines möglichen Libyen-Einsatzes der Bundeswehr darauf zurückgeführt wird, wie dieser Einsatz zuvor dargestellt wurde. So werden auf der Mikroebene die Effekte einer Kommunikationsstrategie isoliert, die die humanitäre Zielsetzung des diskutierten Einsatzes in den Vordergrund und damit dessen kriegerischen Aspekt in den Hintergrund rückt. Mit Hilfe von Interaktionstermen wird zudem analysiert, ob alle Bürger in gleichem Maße auf eine derartige Kommunikationsstrategie reagieren oder ob bestimmte Bevölkerungsgruppen für diese Strategien besonders empfänglich sind. Im Folgenden wird zunächst die Entwicklung der politischen Kommunikation über die Beteiligung der Bundeswehr an multilateral geführten militärischen Auslandseinsätzen dargestellt. Danach werden die theoretischen Mechanismen diskutiert, wie Kommunikationsstrategien auf der Individualebene zu Mei-

3 Exzellente Überblicke über den theoretischen und empirischen Forschungsstand liefern Chong/Druckman (2007a; 2007b).

4 Siehe hierzu die Literatur zur strategischen Kultur wie etwa bei Berger (1998), Duffield (1998), Göler (2010), Wittlinger (2010) und zu den Bevölkerungseinstellungen zur Außenund Sicherheitspolitik in Deutschland wie etwa bei Asmus et al. (2005), Biehl et al. (2011), Fiebig (2008), Isernia/Everts (2004), Juhász (1996; 2001). 
nungsverschiebungen führen. ${ }^{5}$ Im Anschluss wird der Libyen-Fall genauer vorgestellt, der zur Überprüfung der Wirksamkeit von Kommunikationsstrategien herangezogen werden soll. Vor allem der Einsatztyp und dessen Darstellung in der öffentlichen Debatte stellen hierbei wichtige Kontextfaktoren dar, die beeinflussen, wie die Bürger den Libyen-Fall vor der Durchführung des Experiments wahrnehmen und beurteilen. Die anschließende Formulierung von Hypothesen über die Wirkung von Kommunikationsstrategien auf der Individualebene kann daher nur vor dem Hintergrund des im Libyen-Fall vorliegenden spezifischen Kontexts erfolgen. Im Anschluss werden die Hypothesen empirisch untersucht. Der Aufsatz schließt mit der Diskussion und Einordnung der Befunde.

\section{Auslandseinsätze, öffentlicher Diskurs und Bevölkerungsurteile}

Um die Beteiligung der Bundeswehr an zunehmend umfangreichen und intensiven Auslandsmissionen zu rechtfertigen und um für öffentliche Unterstützung dieser Maßnahmen zu werben, haben politische Entscheidungsträger unterschiedliche Aspekte der Einsätze in den Vordergrund gerückt (Baumann/Hellmann 2001: 78). So wurden in den Bundestagsdebatten und Regierungserklärungen neben der Verantwortung gegenüber der internationalen Gemeinschaft und der Notwendigkeit einer verlässlichen, multilateral orientierten deutschen Außenpolitik häufig humanitäre Überlegungen als Argumente für solche Missionen angeführt. ${ }^{6}$ Gleichzeitig vermieden die politischen Entscheidungsträger den expliziten Verweis auf den militärischen Charakter der Auslandseinsätze (Müller et al. 2010: 21; Schörnig 2009: 1). Nach Ansicht von Harald Müller und Koautoren handelte es sich hierbei um »Verdrängung und Vermeidungsrhetorik« (Müller et al. 2010: 20), mit der eine offene Debatte über die Gründe und Ziele der Auslandseinsätze umgangen werden sollte. Insgesamt lässt sich somit eine Doppelstrategie identifizieren, mit der politische Entscheidungsträger versucht haben, öffentliche Unterstützung für die Beteiligung der Bundeswehr an Auslandseinsätzen zu gewinnen. Zum einen wurden unterschiedliche Ziele genannt, gegenüber denen die Deutschen aufgeschlossen sind, zum anderen wurde der militärische Charakter - und die damit einhergehenden Gefahren und Risiken für die Soldaten - heruntergespielt.

5 Um die momentane Gesamtbewertung eines Objekts (Eagly/Chaiken 1993: 1; Fazio 2007: 608) zu bezeichnen, hier die Entsendung deutscher Soldaten in einen Libyen-Einsatz, verwenden wir die Ausdrücke Urteil, Bewertung und Meinung synonym.

6 In den Diskursen um die Teilnahme an Auslandseinsätzen spielten zudem rechtliche Aspekte eine zentrale Rolle. So wurde bis zum Urteil des Bundesverfassungsgericht 1994 diskutiert, ob Auslandseinsätze der Bundeswehr überhaupt verfassungsrechtlich zulässig sind (Philippi 1997: 46-47). Bei der Teilnahme der Bundeswehr am Kosovokrieg stand die fehlende völkerrechtliche Legitimation durch ein UN-Mandat im Zentrum der Debatte und führte bei den Grünen zu einer innerparteilichen Zerreißprobe (Maull 2000: 62). Da dieser Aspekt bei dem Libyen-Einsatz jedoch keine Rolle spielte, kann er hier vernachlässigt werden. 
Die Analyse des Zusammenhangs von Kommunikationsstrategien und Bevölkerungsbewertungen ist Gegenstand der Forschung zu sogenannten framing-Effekten. ${ }^{7}$ Die Einbettung eines politischen Problems in einen bestimmten Kontext wird hier als Frame bezeichnet. Politische Akteure präsentieren den Bürgern Sachfragen stets vor dem Hintergrund von Problembeschreibungen, Ursache-Wirkungs-Zusammenhängen und moralischen Überlegungen. Dieser Kommunikationsprozess, in dem die Kontextualisierung des fraglichen Gegenstandes erfolgt und eine bestimmte Sichtweise auf den Gegenstand etabliert wird, kann dann als framing verstanden werden. Framing kann den kognitiven Prozess beeinflussen, in dem die Auswahl und Gewichtung jener Überlegungen erfolgt, die eine Person berücksichtigt, wenn sie sich ein Urteil über einen Gegenstand bildet. ${ }^{8}$ Es fließen also nicht völlig neue, noch nie gehörte Überlegungen in die Urteilsbildung ein, vielmehr werden bereits im Langzeitgedächtnis gespeicherte, zuvor nicht oder wenig berücksichtigte Aspekte reaktiviert und verstärkt. Sprechen die Frames Aspekte an, die keine weiteren Überlegungen reaktivieren oder mit denen die Bürger völlig unvertraut sind, ergeben sich dagegen keine Bewertungsverschiebungen (Chong/Druckman 2007a: 110-111; 2007b: 107; Druckman/Leeper 2012: 876).

$\mathrm{Ob}$ und wie stark spezifische Kommunikationsstrategien die Zustimmung zu einem bestimmten Einsatz beeinflussen, hängt also von den Überlegungen ab, die Bürger anstellen, wenn sie sich zu Auslandseinsätzen eine Meinung bilden. Um die Mechanismen genauer untersuchen zu können, die der (Un-)Wirksamkeit von Kommunikationsstrategien zugrunde liegen, müssen diese Überlegungen folglich hier berücksichtigt werden. Die Forschung zu außen- und sicherheitspolitischen Orientierungen hat eine Reihe individueller Merkmale identifiziert, die beeinflussen, welche Überlegungen Bürger bei der Meinungsbildung anstellen. Bei diesen Merkmalen wird davon ausgegangen, dass sie zumindest teilweise bereits während der politischen Sozialisationsphase erworben wurden und daher sowohl relativ stabil als auch relevant für die Meinungsbildung über spezifische Sachfragen sind (Campbell et al. 1960: 126; Feldman 1988: 418; Zaller 1992: 34).

Als Erstes sind dabei bereichsspezifische Grundhaltungen zu nennen, die als allgemeine Maßstäbe der Beurteilung konkreter politischer Sachfragen dienen (Hur-

7 Die Forschung hat eine große Anzahl an Belegen dafür vorgelegt, dass framing Bevölkerungsbewertungen verschieben kann. Wichtige Beiträge sind etwa Chong/Druckman (2010), Druckman (2001a; 2001b); Nelson/Kinder (1996); Nelson et al. (1997) und Sniderman/Theriault (2004).

8 Unter Überlegungen sind hier Gründe zu verstehen, die zur Zustimmung oder Ablehnung einer (politischen) Position führen können. Sie sind somit »a compound of cognition and affect - that is, a belief about an object and an evaluation of the belief« (Zaller 1992: 40). Formal ausgedrückt kann eine individuelle Gesamtbewertung (A) in diesem Kontext als Summe einer Menge (i) an Überlegungen (v) aufgefasst werden, die eine Person mit dem Einstellungsobjekt verbindet und entsprechend ihrer subjektiven Bedeutung (w) bei der Meinungsbildung berücksichtigt: $A=\sum \mathrm{v}(\mathrm{i}) * \mathrm{w}(\mathrm{i})$ (Chong/Druckman 2007b: 107; siehe klassisch Fishbein/Ajzen 1975). 
witz/Peffley 1987: 63). ${ }^{9}$ Für die Außen- und Sicherheitspolitik werden vor allem drei Grundhaltungen diskutiert, die den spezifischeren Meinungen der Bürger Struktur verleihen und von denen zwei hier bereits angesprochen wurden. ${ }^{10}$ Die Erste betrifft das Ausmaß der internationalen Involvierung (Isolationismus - Internationalismus). Während Isolationisten eine starke Involvierung prinzipiell ablehnen, befürworten Internationalisten eine aktive Herangehensweise an internationale Probleme. Die Zweite betrifft die präferierten Mittel der Außenpolitik (Pazifismus - Militarismus). Pazifistisch orientierte Bürger lehnen den Einsatz militärischer Mittel kategorisch ab, während Militaristen diesem Instrument prinzipiell positiv gegenüberstehen. Schließlich geht es um das Verhältnis zu anderen Akteuren des internationalen Systems (Unilateralismus - Multilateralismus). Unilateralisten lehnen die Kooperation mit anderen Akteuren ab, während Multilateralisten die Zusammenarbeit mit anderen Akteuren begrüßen. Für die europäischen Länder haben Ronald Asmus et al. (2005: 403-404) eine weitere, quer zum Multilateralismus gelagerte Grundhaltung vorgeschlagen, die auf die Zusammenarbeit mit dem transatlantischen Partner abzielt (Atlantizismus). Bürger mit geringer transatlantischer Orientierung stehen dabei jeglichen transatlantischen Koordinationsbemühungen skeptisch gegenüber, während eine starke transatlantische Orientierung die Bereitschaft impliziert, US-amerikanischer Führung zu folgen und die eigene Außenpolitik entsprechend auszurichten.

Neben diesen bereichsspezifischen Grundhaltungen sollen mit Parteiidentifikation und Links-rechts-Orientierung Dispositionen berücksichtigt werden, die thematisch nicht unmittelbar mit der Außen- und Sicherheitspolitik verbunden sind. Die politische Kommunikation über beinahe alle Sachfragen wird jedoch entlang dieser - teilweise überlappenden - Konfliktlinien geführt, weshalb sie die Urteilsbildung zur Außen- und Sicherheitspolitik ebenfalls beeinflussen (Holsti 2004: 232; Juhász 1996: 121; Juház 2001: 80). Parteibindungen sind vor dem Hintergrund der elite cueing-Heuristik und der Wirkungen sozialer Identitäten relevant für die Meinungsbildung (Green et al. 2002: 8; Zaller 1992: 287). Dabei übernehmen Bürger die Positionen jener Politiker, denen sie vertrauen. Dieser Zusammenhang kann zum einen als Heuristik interpretiert werden, die von den Bürgern mehr oder weniger bewusst verwendet wird, um Informationskosten zu sparen (Kuklinski/Hurley 1994: 730; Lupia 1992: 398). Alternativ können Parteibindungen als eine soziale Identität

9 Auf gleiche Weise können Wertorientierungen zur Meinungsbildung herangezogen werden (Feldman 1988: 418; Nincic/Ramos 2010: 120). Im vorliegenden Fall dürften außenund sicherheitspolitische Grundhaltungen sowie die unten diskutierten Parteiidentifikationen und ideologischen Positionen unter anderem Wertekomponenten enthalten (Schwartz et al. 2010: 422; siehe auch Pötzschke et al. 2013).

10 Vgl. etwa Chittick et al. (1995); Holsti (2004); Rathbun (2007). Die folgenden Begriffspaare beziehen sich jeweils auf die Extrempositionen eines Kontinuums, auf dem die Grundhaltungen der Bürger jeweils angeordnet werden können. Wenn im Folgenden beispielsweise davon gesprochen wird, dass einige Bürger militaristischere Grundhaltungen aufweisen als andere, ist damit gemeint, dass Erstere näher an der Extremposition Militarismus positioniert sind als Letztere. 
interpretiert werden, mit der ein psychologisches Bedürfnis zu gruppenkonformem Verhalten einhergeht (Greene 1999: 394; Tajfel/Turner 1979: 40; Turner 1982: 31).

Die Links-rechts-Dimension kann als super issue verstanden werden, das sowohl Bürger als auch politische Eliten benutzen, um die Komplexität politischer Inhalte und Prozesse zu reduzieren (Arzheimer 2009: 97; Mair 2007: 206). Wie die Parteibindung kann auch die Selbstplatzierung in das linke bzw. rechte Lager als Informationskosten sparende Heuristik interpretiert werden oder ist Ausdruck einer sozialen Identität, die mit dem bereits diskutierten Konformitätsbedürfnis einhergeht. Damit ist jedoch nicht gesagt, dass alle Bürger bei jedem Thema und zu jeder Zeit eine policy-Position aus ihrer Links-rechts-Orientierung ableiten können. Notwendige Bedingung dafür ist, dass die Links-rechts-Dimension Teil des öffentlichen Diskurses zu dem fraglichen Thema ist. Gleichzeitig kann die Selbstplatzierung auf dieser Dimension als Ausdruck einer bestimmten Kombination universeller Werte verstanden werden. Linke sind meist universalistischer und benevolenter eingestellt, während Rechte stärker macht- und leistungsorientiert sind und ein größeres Sicherheitsbedürfnis aufweisen (Caprara et al. 2006: 20; Schwartz et al. 2010: 429-430; Thórisdóttir et al. 2007: 180-181).

Damit sind die individuellen Merkmale vorgestellt, die die Bewertungen militärischer Auslandseinsätze beeinflussen und gleichzeitig dafür sorgen sollten, dass unterschiedliche Darstellungen dieser Sachfrage zu unterschiedlich starken Verschiebungen im Zustimmungsniveau führen. Im nächsten Schritt beschreiben wir den Libyen-Fall und das Umfrageexperiment, mit dem die Wirkung von Kommunikationsstrategien untersucht wird. Vor diesem Hintergrund werden aus den allgemeinen theoretischen Überlegungen dann spezifische Hypothesen formuliert.

\section{Der Libyen-Fall: Untersuchungsanlage und Hypothesen}

Bei dem Libyen-Einsatz war auf Seiten der Regierung eine Kommunikationsstrategie zu beobachten, die bekannte Motive vorangegangener Diskussionen aufgriff. Die Enthaltung der schwarz-gelben Bundesregierung bei der Abstimmung im Sicherheitsrat über Resolution 1973, die den Einsatz militärischer Mittel zur Durchsetzung einer Flugverbotszone im libyschen Luftraum autorisierte, wurde damit begründet, dass Deutschland bzw. die Bundeswehr bei einer Zustimmung in den Konflikt hineingezogen worden wäre. Nach Ansicht der Bundesregierung musste vermieden werden, auf eine »schiefe Ebene« (Westerwelle 2011: 10816) zu geraten, die aufgrund der Unvorhersehbarkeit militärischer Auseinandersetzungen leicht in der Entsendung von Bodentruppen enden könne (Merkel 2011: 11251). Insgesamt wurden also die Risiken und Unwägbarkeiten derartiger militärischer Interventionen hervorgehoben. So warnte der deutsche Botschafter bei den Vereinten Nationen in seiner Begründung des deutschen Abstimmungsverhaltens davor, die Wahrscheinlichkeit zahlreicher Todesopfer zu unterschätzen (Wittig 2011: 5). Vor diesem Hintergrund wurde das humanitäre Ziel des UN-Mandats, nämlich Muammar al-Gaddafi daran zu hindern, das Militär (weiter) gegen das eigene Volk einzuset- 
zen und nach der erfolgreichen Niederschlagung der Rebellion Rache an den Aufständischen zu nehmen, weder verschwiegen noch diskreditiert, im Vergleich zu den unsicheren Erfolgsaussichten und großen Risiken jedoch in den Hintergrund gedrängt (Hellmann 2011: 21; Maull 2011: 107-108; Müller 2011: 6).

Um die Wirkung unterschiedlicher Darstellungen des möglichen Einsatzes der Bundeswehr in Libyen auf die Meinungsbildung zu dieser Sachfrage zu untersuchen, ist das Instrument des Umfrageexperiments besonders gut geeignet. Der entscheidende Vorteil des experimentellen Designs liegt dabei in der Möglichkeit, durch die zufällige Einteilung der Beobachtungseinheiten in Experimental- und Kontrollgruppen sowie die kontrollierte Manipulation einer interessierenden Variable in der einen Gruppe Inferenzprobleme zu umgehen, die bei der Analyse reiner Beobachtungsdaten auftreten. Gelingen die Randomisierung der Gruppeneinteilung sowie die kontrollierte und standardisierte Manipulation einer Variable in der Experimentalgruppe, kann das Experiment insofern als intern valide gelten, als Unterschiede in der Ausprägung der abhängigen Variablen in Experimental- und Kontrollgruppe kausal auf die manipulierte unabhängige Variable zurückgeführt werden können (McDermott 2011a: 28). ${ }^{11}$

Bei dem framing-Experiment, das die Grundlage dieser Analyse darstellt, wurden die Teilnehmer des GLES-Online-Trackings (T14), das vom 23.5.2011 bis 3.6.2011 durchgeführt wurde, über ihre Meinung zu einer möglichen Beteiligung der Bundeswehr an Einsätzen in Libyen befragt $(n=1154) .{ }^{12}$ Dabei wurden die Befragten zufällig einer von drei Gruppen zugewiesen und jeweils unterschiedlich an die Frage nach ihrer Unterstützung einer deutschen Beteiligung herangeführt. In allen drei Gruppen wurden die Befragten zunächst darauf hingewiesen, dass die Möglichkeit solcher Einsätze in Libyen sowohl in Deutschland als auch in Europa diskutiert werde. Die Personen in der ersten Gruppe wurden daraufhin lediglich gefragt, ob sie einer Beteiligung deutscher Soldaten an solchen militärischen Einsätzen zustimmen würden. Der zweiten und dritten Gruppe wurde die Frage dagegen vor dem Hintergrund jeweils unterschiedlicher Problemkontexte präsentiert. In der zweiten Version wurde darauf hingewiesen, dass bei einem solchen Libyen-Einsatz natürlich auch die Gefahr bestehe, dass die Soldaten in Kampfhandlungen verwickelt werden. Diese Darstellung zielt also auf die hohe Konfliktintensität, die mit diesem

11 Experimente und vor allem Umfrageexperimente stellen in der Politikwissenschaft zunehmend wichtige Instrumente für die Analyse von Kausalzusammenhängen dar. Siehe hierzu beispielsweise Druckman et al. (2011). Speziell für den Bereich der Internationalen Beziehungen wird dieses Instrument beispielsweise in Hermann/Ozkececi-Taner (2011) und in dem von McDermott und Mintz organisierten Symposium der International Studies Quarterly (2011, 55: 2) diskutiert. Siehe vor allem McDermott (2011b) und Mintz et al. (2011).

12 Bei den Online-Trackings handelt es sich um eine im Auftrag der German Longitudinal Election Study (GLES) vierteljährlich wiederholte Befragung zufällig ausgewählter Mitglieder des Online-Access-Panels der Respondi AG, das 2011 etwa 100.000 aktive Panelisten umfasste (für weitere Informationen siehe Rattinger et al. 2011). Wir danken der Gesellschaft für Wahlforschung (DGfW) und den Primärforschern der German Longitudinal Election Study (GLES) für die Aufnahme des framing-Experiments in diese Onlinebefragung. 
politischen Mittel einhergeht (Mittel-Frame). In der dritten Version wurde dagegen betont, dass bei diesen Einsätzen beispielsweise Flüchtlinge in Sicherheit gebracht werden sollten. Dieser Frame bezieht sich folglich auf die humanitäre Zielsetzung (Ziel-Frame). Im Anschluss wurde jeweils - wie bei der Gruppe mit dem neutralen Frame - nach der Zustimmung zu einer deutschen Beteiligung an derartigen Einsätzen gefragt. ${ }^{13}$

Da das Experiment im Rahmen einer Online-Befragung der Mitglieder eines Online-Access-Panels durchgeführt wurde, stellen die Teilnehmer keine repräsentative Stichprobe der Gesamtbevölkerung dar (Faas/Schoen 2006: 178; Sanders et al. 2007: 260). Allerdings unterscheidet sich unsere Stichprobe im Hinblick auf Merkmale, die den Umfang von framing-Effekten beeinflussen könnten, also etwa der formalen Bildung, dem politischen Interesse und der Links-rechts-Orientierung (Druckman/Nelson 2003: 731-732; Miller/Krosnick 2000: 303), nicht wesentlich von den Befragten, die vom 24. bis zum 26. Mai 2011 im Rahmen der Politbarometer-Erhebungen telefonisch interviewt wurden. Merkliche Unterschiede treten hinsichtlich des Lebensalters und der Parteiidentifikation auf, doch haben diese Merkmale - wie unten gezeigt wird - offenbar keinen Einfluss auf die framing-Effekte. Daher dürfte die Stichprobenzusammensetzung insofern zu keinen gravierenden Verzerrungen führen. ${ }^{14}$

Hinsichtlich der externen Validität der Befunde ist auch zu bedenken, dass sich die Bedingungen in unserem framing-Experiment im Hinblick auf die Darbietung und den Inhalt der Frames von der realen Situation unterscheiden. Reale politische Diskurse erstrecken sich typischerweise über einen längeren Zeitraum, in dem Politiker Bürgern die von ihnen vertretenen Positionen wiederholt mitteilen und auf die Gegenseite eingehen können. Die einmalige Konfrontation mit nur einer spezifischen Darstellung der Libyen-Frage bildet diese Situation nur ansatzweise ab. ${ }^{15} \mathrm{Da}-$ her können unsere Befunde, etwa zu Effektstärken, nicht unbesehen auf die reale gesamtgesellschaftliche politische Urteilsbildung übertragen werden. Mit dem vor-

13 Für die genaue Frageformulierung der Experimentalbedingungen sowie aller weiterer Operationalisierungen siehe den Anhang, der auch die Randverteilungen der verwendeten Variablen enthält. Bei der Messung der Grundhaltungen zum Multilateralismus und Atlantizismus wurden Items verwendet, die jeweils unterschiedliche Subdimensionen der theoretischen Konzepte erfassen sollen. Maße der internen Konsistenz wie Cronbachs Alpha fallen daher relativ niedrig aus $(0,27$ bzw. 0,33$)$.

14 Die Ergebnisse des Stichprobenvergleichs sind bei den Autoren erhältlich.

15 Sniderman/Theriault (2004: 146) weisen beispielsweise darauf hin, dass Bürger typischerweise gleichzeitig mit mehreren, rivalisierenden Sichtweisen konfrontiert werden, was die Wirksamkeit eines einzelnen Frames beeinflussen kann. Ebenfalls von Bedeutung ist die Glaubwürdigkeit der Person, die den Frame kommuniziert (Baum/Groeling 2009: 27; Druckman 2001b: 1045). Die Wirkung einer einmaligen Konfrontation mit einem Frame lässt zudem im Zeitverlauf nach, was besonders dann der Fall ist, wenn Bürger nicht durch eigenständige Informationssuche auf einen Frame stoßen, sondern mit diesem - etwa über die Massenmedien - konfrontiert werden (Chong/Druckman 2010: 665; Druckman et al. 2012: 433). Auch die Möglichkeit, mit Familie und Bekannten über ein Thema zu sprechen, kann die Wirkung von Frames reduzieren, die politische Entscheidungsträger über die Massenmedien kommunizieren (Druckman 2004: 675; Druckman/Nelson 2003: 730). 
liegenden Umfrageexperiment verfolgen wir deshalb das - bescheidene - Ziel, erstmals methodisch angemessen zu untersuchen, ob es möglich ist, die Bewertungen von Bürgern zu Auslandseinsätzen der Bundeswehr trotz ihrer allgemein kritischen Haltung zum Einsatz militärischer Mittel mithilfe von Kommunikationsstrategien zu verschieben. Um weitergehende Folgerungen über die Wirkungen von Kommunikationsstrategien abzuleiten, wären anspruchsvollere Untersuchungsdesigns bis hin zu großen Feldexperimenten erforderlich, die es erlauben, kausale Effekte in realweltlichen Kontexten zu isolieren (z.B. Gerber et al. 2011).

Im Folgenden sollen spezifische Hypothesen zur Wirksamkeit der Frames formuliert werden (siehe für einen Überblick Tabelle 1). Dies kann nur unter Berücksichtigung der Kontextbedingungen geschehen, die vor der Durchführung des Experiments gegeben waren (pretreatment environment) (Druckman/Leeper 2012: 876). $\mathrm{Da}$ in der Diskussion über die mögliche Entsendung der Bundeswehr nach Libyen wie oben dargestellt - kein Hehl daraus gemacht wurde, dass es sich hierbei um einen Kampfeinsatz handeln würde, sollte die Konfrontation mit dem Mittel-Frame im Vergleich zu der neutralen Darstellung zu keiner stärkeren Ablehnung des Einsatzes führen. Auch ohne den expliziten Verweis des Frames auf die drohenden militärischen Auseinandersetzungen dürften den Bürger mehrheitlich diese Assoziationen durch den Kopf gehen. Dagegen sollte vom Ziel-Frame ein starker Effekt ausgehen. Das moralisch wertvolle Ziel, Unschuldigen zu helfen, dürfte bei den meisten Befragten ohne expliziten Hinweis keine oder nur eine geringe Rolle bei der Meinungsbildung spielen, da der Diskurs in Deutschland vor allem um die Politik der Bundesregierung und die prinzipielle Rechtfertigung des Eingreifens in einen Bürgerkrieg und der - implizit angestrebten - Absetzung eines diktatorischen Machthabers kreiste. ${ }^{16}$

Inwiefern die Bürger in unterschiedlichem Maße für die Kommunikationsstrategie empfänglich sind, die den möglichen Libyen-Einsatz als humanitäre Intervention darstellt, soll in einem zweiten Schritt untersucht werden, für den nun ebenfalls konkrete Erwartungen formuliert werden. Verschiebungen sind vor allem aufgrund jener Grundhaltungen zu erwarten, die die Akzeptanz des genannten Ziels - die Rettung von Flüchtlingen - beeinflussen. Da diese Darstellung vom Instrumententyp (militärischer Kampfeinsatz) ablenkt und die Zieldimension in den Mittelpunkt rückt, dürfte die Grundhaltung zur Einmischung in internationale Angelegenheiten die Effektstärke des Frames beeinflussen. Personen, die die Verfolgung außenpolitischer Ziele prinzipiell eher ablehnen und der Meinung sind, dass die Regierung Zeit und Geld besser zur Lösung innenpolitischer Probleme verwenden sollte, dürften durch den Verweis auf die humanitäre Zielsetzung weit weniger beeinflusst werden als Personen mit einer Präferenz für eine aktive deutsche Außenpolitik. Für Erstere spielt die Zieldimension bei der Bewertung außen- und sicherheitspoliti-

16 Andere Rahmenbedingungen implizieren dagegen andere Hypothesen. Hätten die außenund sicherheitspolitischen Eliten die Risiken eines Kampfeinsatzes in Libyen heruntergespielt und die humanitäre Zielsetzung betont, wären die Erwartungen bezüglich der Wirksamkeit der beiden Frames annähernd umgekehrt. Der Ziel-Frame sollte dann keinen Effekt haben, der Mittel-Frame dagegen zu einer Reduktion der Unterstützung führen. 
scher Maßnahmen keine Rolle, da sie die Verfolgung solcher Ziele prinzipiell ablehnen. Der explizite Verweis auf ein außen- und sicherheitspolitisches Ziel - und sei es moralisch noch so unproblematisch - sollte bei ihnen also keinen Zustimmungszuwachs auslösen.

Auch die Links-rechts-Orientierung sollte eine Rolle dabei spielen, wie sehr der Verweis auf die humanitären Ziele zu einer positiveren Bewertung einer deutschen Beteiligung führt. Da Personen, die sich weiter rechts auf der Links-rechts-Dimension platzieren, weniger inklusive Solidaritätsvorstellungen hegen und der Wohlfahrt der Menschen anderer Länder einen geringeren Stellenwert zuweisen als Linke (Schwartz et al. 2010: 429-430; Thórisdóttir et al. 2007: 180-181), sollte der Effekt des Ziel-Frames bei Letzteren größer sein als bei Ersteren. Im Gegensatz zu der zuvor formulierten Erwartung entstehen die Unterschiede in den Effektstärken also nicht daraus, dass außen- und sicherheitspolitische Ziele als Bewertungsmaßstab prinzipiell akzeptiert oder - von Isolationisten - grundsätzlich abgelehnt werden. Entscheidend ist, wie wichtig und wünschenswert ein fragliches Ziel aus Sicht der Bürger inhaltlich ist.

Für die übrigen Grundhaltungen und die Identifikation mit einer der Regierungsparteien sind dagegen keine Interaktionseffekte zu erwarten. Die allgemeinen Vorstellungen zum Einsatz militärischer Mittel sollten die Wirksamkeit des ZielFrames nicht beeinflussen, da sie unabhängig von der humanitären Dimension sind, die der Frame anspricht. Gleiches gilt für die Präferenzen darüber, ob die Bundesrepublik in der Außen- und Sicherheitspolitik mit anderen Ländern kooperieren soll die Grundhaltungen zu Multilateralismus und Atlantizismus. Auch die Identifikation mit einer der Regierungsparteien sollte die Wirksamkeit des Frames nicht beeinflussen. Zwar legt die humanitäre Darstellung einen anderen Schluss in Bezug auf die Entsendung der Bundeswehr nahe, als ihn die Bundesregierung gezogen hat, was bei deren Parteianhängern zu einer abwehrenden Reaktion auf den Ziel-Frame führen könnte. Diese Position war innerhalb des Regierungslagers allerdings nicht unumstritten, so dass deren Mitglieder nicht geschlossen für sie warben. Zudem nutzten die anderen Parteien den humanitären Frame nicht, um für eine deutsche Beteiligung zu werben, so dass diese Darstellung insgesamt bei Regierungsanhängern nicht anders gewirkt haben dürfte als bei den übrigen Bürgern. 
Tabelle 1: Überblick Hypothesen: (Bedingte) Effekte der Frames auf die Unterstützung eines möglichen Einsatzes der Bundeswehr in Libyen

\begin{tabular}{ll}
\hline & $\begin{array}{l}\text { Unterstützung eines möglichen Bundeswehreinsatzes in Li- } \\
\text { byen }\end{array}$ \\
\hline Ziel-Frame & + \\
Mittel-Frame & Kein Effekt \\
Ziel-Frame * Militarismus & Kein Effekt \\
Ziel-Frame * Internationalismus & + \\
Ziel-Frame * Multilateralismus & Kein Effekt \\
Ziel-Frame * Atlantizismus & Kein Effekt \\
Ziel-Frame* Links-rechts-Orientierung & - \\
Ziel-Frame* PID Regierung & Kein Effekt \\
\hline
\end{tabular}

\section{Ergebnisse}

Entscheidend für die interne Validität der weiteren Analysen ist, dass sich die Teilnehmer des Umfrageexperiments in allen Gruppen im Hinblick auf theoretisch relevante Merkmale ähneln. ${ }^{17}$ Nur dann ist es möglich, Unterschiede in den Bewertungen des möglichen Libyen-Einsatzes tatsächlich auf die unterschiedlichen Frames zurückzuführen (interne Validität). Tabellen 2 und 3 zeigen, dass dies für soziodemografische Hintergrundvariablen und die im vorherigen Abschnitt diskutierten, potenziellen Erklärungsfaktoren außen- und sicherheitspolitischer Meinungen (bis auf eine Ausnahme) tatsächlich der Fall ist. Lediglich das durchschnittliche Interesse für Außen- und Sicherheitspolitik weicht statistisch signifikant von einer Gleichverteilung ab. Die Befragten, die die neutrale Frage zum möglichen LibyenEinsatz gestellt bekamen, sind im Vergleich zu den Befragten der anderen beiden Gruppen politisch etwas weniger interessiert. Allerdings fallen diese Unterschiede substanziell kaum ins Gewicht, so dass eine inhaltlich sinnvolle Analyse der framing-Effekte möglich ist. ${ }^{18}$

17 Darüber hinaus lassen black box-Experimente nur dann Schlüsse auf kausale Prozesse zu, wenn eine Reihe weiterer Bedingungen erfüllt sind (Green et al. 2010: 202-203; Imai et al. 2011: 770-772). Da es mit den vorliegenden Daten nicht möglich ist zu überprüfen, ob dies hier der Fall ist, ist der Schluss von den präsentierten Ergebnissen auf tatsächliche Kausalprozesse aus streng methodischer Sicht nicht unproblematisch.

18 Da Signifikanztests in diesem Kontext nicht unumstritten sind (Ho et al. 2007: 221; Imai et al. 2008: 495-496), wurde zusätzlich jeweils das Verhältnis von durchschnittlichem Gruppenunterschied und Standardabweichung überprüft (Ergebnisse nicht tabellarisch ausgewiesen). Diese sowie alle weiteren hier nicht tabellarisch ausgewiesenen Analysen sind auf Anfrage bei den Autoren erhältlich. Bei allen Variablen liegt das Verhältnis deutlich unter einem Viertel, was für die Gleichverteilung der Merkmale in den Experimentalgruppen spricht (Imai et al. 2008: 498). 
Tabelle 2: Teilnehmerzahl und Sozialstruktur nach Fragebogenversion

\begin{tabular}{lcccccc}
\hline Version & $\begin{array}{c}\text { Anteil Teil- } \\
\text { nehmer }\end{array}$ & $\begin{array}{c}\text { Mittleres } \\
\text { Alter }\end{array}$ & $\begin{array}{c}\text { Anteil Abitu- } \\
\text { rienten }\end{array}$ & $\begin{array}{c}\text { Anteil } \\
\text { Frauen }\end{array}$ & $\begin{array}{c}\text { Anteil Ost- } \\
\text { deutsche }\end{array}$ & $\begin{array}{c}\text { Durch- } \\
\text { schnittl.Interes- } \\
\text { se }\end{array}$ \\
\hline Ziel-Frame & 33,3 & 43,0 & 22,6 & 47,1 & 24,7 & 0,50 \\
Neutraler Frame & 33,3 & 43,1 & 16,6 & 52,0 & 25,6 & 0,45 \\
Mittel-Frame & 33,3 & 43,4 & 20,3 & 48,8 & 22,6 & 0,51 \\
\hline F & & 0,1 & 2,2 & 0,9 & 0,5 & $4,2^{*}$ \\
\hline
\end{tabular}

Signifikanzniveaus: ${ }^{* * *} \mathrm{p}<0,001 ;{ }^{* *} \mathrm{p}<0.01 ;{ }^{*} \mathrm{p}<0.05$

Tabelle 3: Theoretisch relevante Variablen nach Fragebogenversion

\begin{tabular}{lcccccc}
\hline Version & $\begin{array}{c}\text { Milita- } \\
\text { rismus }\end{array}$ & $\begin{array}{c}\text { Internatio- } \\
\text { nalismus }\end{array}$ & $\begin{array}{c}\text { Multilatera- } \\
\text { lismus }\end{array}$ & $\begin{array}{c}\text { Atlanti- } \\
\text { zismus }\end{array}$ & $\begin{array}{c}\text { Links- } \\
\text { Rechts }\end{array}$ & \multicolumn{2}{c}{ Regierungs- } \\
PID
\end{tabular}

Signifikanzniveaus: ${ }^{* * *} \mathrm{p}<0,001 ;{ }^{* *} \mathrm{p}<0.01 ;{ }^{*} \mathrm{p}<0.05$

Wertebereich aller Variablen auf $0-1$ gesetzt

Um zu prüfen, ob sich die Zustimmung zu dem möglichen Libyen-Einsatz mit dem Umfrageexperiment in der erwarteten Weise verschieben lässt, vergleichen wir zunächst die durchschnittliche Bewertung eines solchen Einsatzes in den drei Experimentalgruppen. In Tabelle 4 sind die Ergebnisse dieser Analyse dargestellt. Wie erwartet zeigt sich ein substanzieller Unterschied zwischen der durchschnittlichen Zustimmungsrate in der Gruppe mit dem Ziel-Frame und jenen der beiden anderen Frageversionen. ${ }^{19}$

19 Der Unterschied in den Zustimmungsniveaus bei neutralem und Mittel-Frame ist statistisch nicht signifikant $(\mathrm{t}=0,217)$. 
Tabelle 4: Unterstützung des möglichen Libyen-Einsatzes nach Treatmentgruppe

\begin{tabular}{lcccc}
\hline Treatmentgruppe & $\mathrm{N}$ & $\begin{array}{c}\text { Durchschnitt- } \\
\text { liche } \\
\text { Unterstützung }\end{array}$ & $\begin{array}{c}\text { Anteil Befragte mit } \\
\text { Indexwert }>0,5\end{array}$ & Standardabweichung \\
\hline Ziel-Frame & 345 & 0,44 & 40,3 & 0,34 \\
Neutraler Frame & 341 & 0,23 & 15,8 & 0,30 \\
Mittel-Frame & 347 & 0,26 & 21,0 & 0,30 \\
\hline
\end{tabular}

Wertebereich der abhängigen Variable auf $0-1$ gesetzt

Wird auf die humanitäre Zielsetzung der diskutierten Einsätze verwiesen, verdoppelt sich die durchschnittliche Zustimmung gegenüber jener bei neutraler Fragestellung von rund 0,2 auf rund 0,4. Wird der militärische Aspekt und die damit verbundene Gefahr von kriegerischen Auseinandersetzungen hervorgehoben, unterscheidet sich die Zustimmung dagegen nicht signifikant vom Antwortverhalten bei der neutralen Fragestellung. ${ }^{20}$ Die höhere durchschnittliche Unterstützung bei humanitärem framing sollte allerdings nicht darüber hinwegtäuschen, dass selbst bei dieser Fragestellung der diskutierte Einsatz der Bundeswehr insgesamt kritisch bewertet wird. $^{21}$

Diese empirischen Befunde rechtfertigen es, die beiden Gruppen mit neutralem und Mittel-Frame zusammenzufassen. Wenn wir im Folgenden die Frage untersuchen, welche individuellen Merkmale dafür verantwortlich sind, dass der ZielFrame die Einsatzunterstützung beeinflusst, vergleichen wir daher Unterschiede zwischen der Befragungsgruppe mit dem Ziel-Frame auf der einen mit den Gruppen mit neutralem und Mittel-Frame auf der anderen Seite. ${ }^{22}$ In einer multivariaten linearen Regressionsanalyse werden die Unterstützung des möglichen Bundeswehreinsatzes in Libyen auf eine Dummy-Variable, die den Ziel-Frame anzeigt, die individuellen Merkmale sowie Interaktionen zwischen diesen beiden Variablen(-

20 Um zu überprüfen, ob die Befragten auch tatsächlich jeweils den gesamten Fragetext und damit den kompletten Frame wahrgenommen haben, wurde die Latenzzeiten der Teilnehmer überprüft (nicht tabellarisch ausgewiesen). Es zeigt sich, dass die Beantwortungszeit bei den beiden längeren Fragen (Ziel- und Mittel-Frame) höher ausfällt. Die Respondenten haben die Frage offenbar ernst genommen und eine wichtige Bedingung für die interne Validität der Ergebnisse somit erfüllt (McDermott 2011a: 28).

21 Substanziell das gleiche Ergebnis ergibt eine Regression des Unterstützungsniveaus aller Befragten auf zwei Dummy-Variablen, die identifizieren, ob ein Befragter die Frage mit Ziel- oder Mittel-Frame erhalten hat (nicht tabellarisch ausgewiesen). Die Dummy-Variable des Ziel-Frames ist hochsignifikant ( $<<0,001$ ), während die Dummy-Variable des Mittel-Frames das konventionelle Signifikanzniveau weit verfehlt $(\mathrm{p}=0,225)$. Um sicherzustellen, dass dieses Ergebnis nicht durch Nonrandomisierung zu Stande gekommen ist, wurde bei der Schätzung dieses Modells zusätzlich für soziodemografische Hintergrundvariablen (Alter, Bildung, Geschlecht, Herkunft) kontrolliert.

22 Um die Legitimität dieses Vorgehens sicherzustellen, wurden die folgenden Analysen zu den Interaktionseffekten zwischen humanitärem Frame und Substanzvariablen analog mit dem militaristischen Frame gerechnet, wobei in letzterem Fall die Referenzgruppe Personen mit neutralem Frame darstellten (nicht tabellarisch ausgewiesen). Es zeigten sich keine statistisch signifikanten und substanziell bedeutenden Interaktionseffekte. 
gruppen), zurückgeführt. ${ }^{23}$ Die Berücksichtigung solcher Interaktionsterme erlaubt es, die Effekte einer unabhängigen Variablen in Abhängigkeit der Ausprägung anderer unabhängiger Variablen zu schätzen (Jaccard et al. 1990: 20-33). Da wir uns im vorliegenden Fall dafür interessieren, ob der Ziel-Frame in verschiedenen Bevölkerungsgruppen in gleichem Maße wirkt, wird hier der Effekt der Dummy-Variable in Abhängigkeit unterschiedlicher Ausprägungen der individuellen Merkmale untersucht. Für diese Berechnung, deren Ergebnisse in Tabelle 5 dargestellt sind, wurden die unabhängigen Variablen zuvor um den Mittelwert zentriert.

Entscheidend im Hinblick auf die vorliegende Fragestellung sind die Ergebnisse zu den Interaktionstermen. Statistisch signifikant sind die Koeffizienten der Interaktion des humanitären Frames mit Internationalismus sowie mit der Links-rechtsOrientierung und zeigen damit an, dass die Wirksamkeit des Frames von der Ausprägung dieser beiden Merkmale abhängt. Grundhaltungen zum Einsatz militärischer Mittel, zu Multilateralismus und Atlantizismus sowie die Identifikation mit einer Regierungspartei spielen für die Wirksamkeit des Ziel-Frames hingegen keine Rolle. Dies entspricht den Erwartungen, stehen diese Merkmale doch in keiner direkten Beziehung zu den Überlegungen, die mit dem Verweis auf die humanitäre Zielsetzung des möglichen Libyen-Einsatzes verbunden sind.

23 Zusätzlich wurden politisches Interesse, Alter, Bildung, Geschlecht sowie Herkunft (Ost-/ Westdeutschland) als Kontrollvariablen in das Modell aufgenommen. Hierbei handelt es sich um Faktoren, die sowohl die hier interessierenden unabhängigen als auch die abhängige Variable beeinflussen und somit für eine verzerrte Schätzung der Koeffizienten berücksichtigt werden müssen. Zur Wirkung dieser Kontrollvariablen auf außen- und sicherheitspolitische Meinungen siehe etwa Juhász (1996), Rattinger (1996), Rattinger/Holst (1998) und Holsti (2004). 
Tabelle 5: Interaktionseffekte zwischen humanitärem Frame und individuellen Merkmalen auf die Unterstützung eines möglichen Bundeswehreinsatzes in Libyen

\begin{tabular}{|c|c|c|}
\hline $\begin{array}{l}\text { Referenz: Mittel-/ } \\
\text { Neutraler Frame }\end{array}$ & $\begin{array}{c}\text { Linearer Effekt } \\
\text { b }\end{array}$ & $\begin{array}{c}\text { Interaktionseffekt } \\
\text { Ziel-Frame * Drittvariablen } \\
\text { b }\end{array}$ \\
\hline Ziel-Frame & $\begin{array}{c}0,18^{* * * *} \\
(0,02)\end{array}$ & \\
\hline \multicolumn{3}{|l|}{ Individuelle Merkmale: } \\
\hline Militarismus & $\begin{array}{c}0,44^{* * * *} \\
(0,04)\end{array}$ & $\begin{array}{l}-0,02 \\
(0,08)\end{array}$ \\
\hline Internationalismus & $\begin{array}{c}0,18^{* * * *} \\
(0,03)\end{array}$ & $\begin{array}{l}0,18^{*} \\
(0,07)\end{array}$ \\
\hline Multilateralismus & $\begin{array}{c}0,24 * * * \\
(0,03)\end{array}$ & $\begin{array}{c}0,10 \\
(0,09)\end{array}$ \\
\hline Atlantizismus & $\begin{array}{l}0,10^{*} \\
(0,05)\end{array}$ & $\begin{array}{l}-0,10 \\
(0,10)\end{array}$ \\
\hline Rechts-links-Orientierung & $\begin{array}{l}-0,10^{*} \\
(0,04)\end{array}$ & $\begin{array}{l}-0,20^{*} \\
(0,09)\end{array}$ \\
\hline Regierungs-PID & $\begin{array}{l}-0,04 \\
(0,02)\end{array}$ & $\begin{array}{l}-0,02 \\
(0,05)\end{array}$ \\
\hline Interesse & $\begin{array}{l}-0,02 \\
(0,04)\end{array}$ & $\begin{array}{l}-0,05 \\
(0,08)\end{array}$ \\
\hline Alter & $\begin{array}{l}-0,05 \\
(0,05)\end{array}$ & $\begin{array}{l}-0,18 \\
(0,11)\end{array}$ \\
\hline Bildung & $\begin{array}{c}0,00 \\
(0,03)\end{array}$ & $\begin{array}{l}-0,02 \\
(0,05)\end{array}$ \\
\hline Geschlecht & $\begin{array}{l}-0,03 \\
(0,02)\end{array}$ & $\begin{array}{l}-0,00 \\
(0,04)\end{array}$ \\
\hline Herkunft & $\begin{array}{l}-0,04 \\
(0,02)\end{array}$ & $\begin{array}{l}-0,10^{*} \\
(0,05)\end{array}$ \\
\hline Konstante & $\begin{array}{c}0,31^{* * *} \\
(0,01)\end{array}$ & \\
\hline
\end{tabular}

$\mathrm{N}=873$; korr. $\mathrm{R}^{2}=0,35$

Signifikanzniveaus: ${ }^{* * *} \mathrm{p}<0,001 ;{ }^{* *} \mathrm{p}<0,01 ;{ }^{*} \mathrm{p}<0,05$

Wertebereich aller Variablen auf $0-1$ gesetzt und um den Mittelwert zentriert 
Abbildung 1: Wirksamkeit des Ziel-Frames auf die Unterstützung eines möglichen Libyen-Einsatzes in Abhängigkeit von sechs individuellen Merkmalen ${ }^{24}$
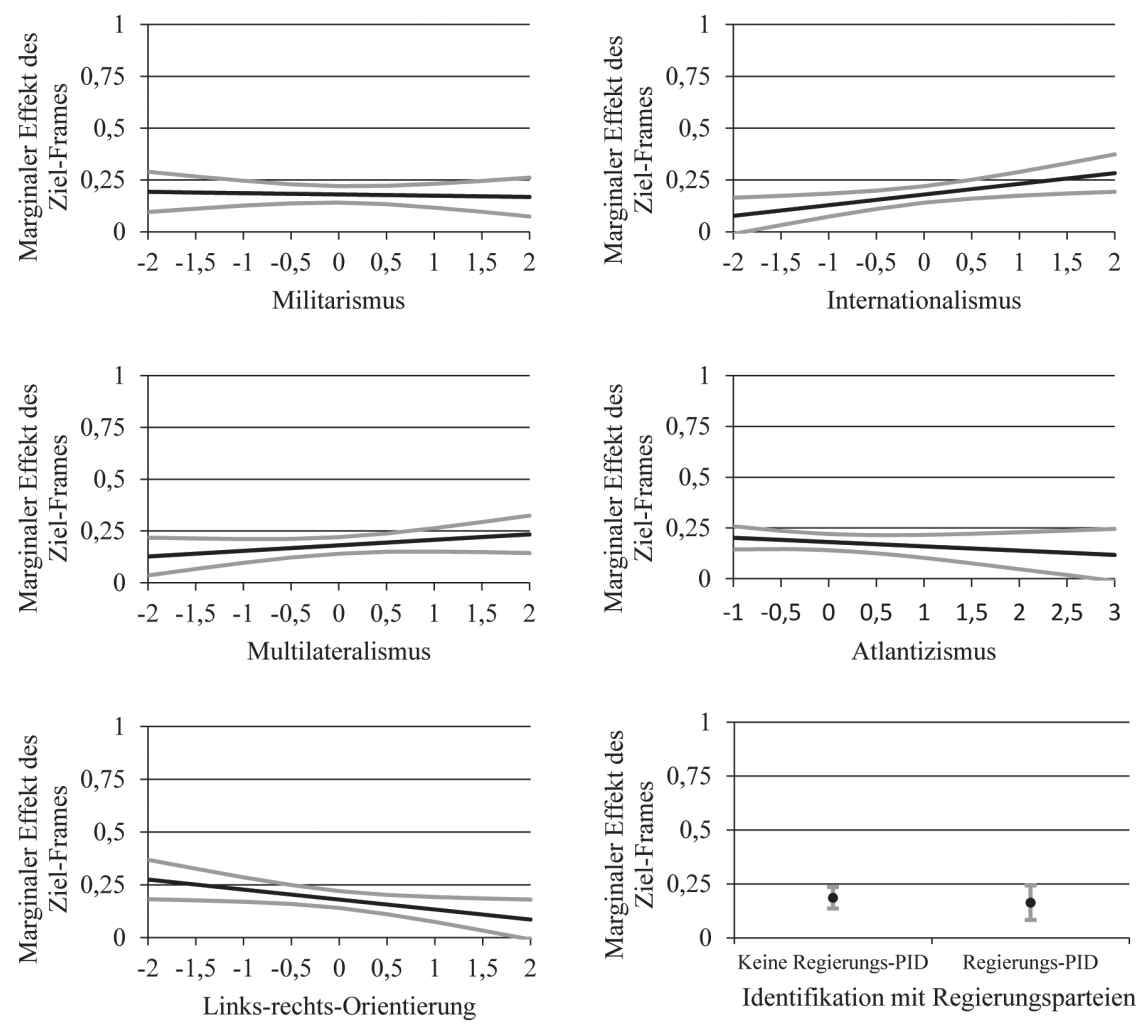

Um die Ergebnisse eingehender zu analysieren, haben wir diese zusätzlich graphisch dargestellt, was die Interpretation erleichtern sollte. Abbildung 1 zeigt die Effekte des Ziel-Frames auf die Einsatzunterstützung (Y-Achse) in Abhängigkeit unterschiedlicher Ausprägungen der individuellen Merkmale (X-Achse) in sechs Koordinatensystemen. ${ }^{25}$ Aufgrund der Zentrierung der unabhängigen Variablen bedeutet ein Wert von null auf der X-Achse, dass die Y-Achse den Effekt des Ziel-

24 Die Ausprägungen der individuellen Merkmale werden in Standardeinheiten angegeben. Die in den Koordinatensystemen abgetragenen marginalen Effekte ergeben sich aus den in Tabelle 5 präsentierten Ergebnissen der Regressionsanalyse und den Standardabweichungen der individuellen Merkmale (nicht tabellarisch ausgewiesen): Koeffizient des Ziel-Frames +/- Standardabweichung(en) des individuellen Merkmals * Koeffizient des Interaktionsterms. Für eine Beispielrechnung siehe Fußnote 27.

25 Die Darstellung des Effekts der Identifikation mit einer der Regierungsparteien weicht von den anderen ab, da dieses Merkmal als kategoriale (Dummy-)Variable operationalisiert wurde. 
Frames bei einer durchschnittlichen Ausprägung sowohl des individuellen Merkmals als auch aller anderer kontrollierter Variablen angibt. ${ }^{26}$ Die Steigungen der Graphen dokumentieren die unterschiedliche Wirksamkeit des Ziel-Frames, wenn die Ausprägungen der individuellen Merkmale über- oder unterdurchschnittlich ausfallen. Eine Einheit auf der X-Achse entspricht dabei einer Standardabweichung, so dass - unter der Annahme einer Normalverteilung - mehr als 95\% der Befragten innerhalb des Wertebereichs von $+/-$ zwei Standardabweichungen um den Mittelwert herum verteilt sind (Kühnel/Krebs 2001: 189). ${ }^{27}$

Die graphische Darstellung veranschaulicht nochmals die Wirkungslosigkeit der Grundhaltungen zum Einsatz militärischer Mittel, zu Multilateralismus und Atlantizismus sowie der Identifikation mit einer der Regierungsparteien. Unterschiede zeigen sich dagegen bei der Grundhaltung zum Internationalismus. Die positive Steigung des Graphen oben rechts in Abbildung 1 macht deutlich, dass Personen, die eine aktive deutsche Außen- und Sicherheitspolitik präferieren, stärker als ihre isolationistischeren Mitbürger durch den Verweis auf die humanitären Ziele zu einer Unterstützung eines möglichen Libyen-Einsatzes bewegt werden. So beträgt der Koeffizient des Ziel-Frames bei Befragten, die sich auf der Internationalismus-Dimension eine Standardabweichung oberhalb des arithmetischen Mittels befinden, 0,23, bei jenen, die eine Standardabweichung unterhalb des Mittelwerts liegen, hingegen lediglich $0,13 .{ }^{28}$ Schon der vergleichsweise holzschnittartige Ziel-Frame führt bei den Befragten demnach dazu, dass sie den humanitären Aspekt des möglichen Libyen-Einsatzes in stärkerem Maße bei der Meinungsbildung berücksichtigen - trotz der allgemein bekannten Risiken und Gefahren, die bei einem derartigen Kampfeinsatz gedroht hätten. Bei extrem isolationistisch orientierten Befragten ist der Effekt des Ziel-Frames nicht mehr statistisch signifikant, wie an der Überlap-

26 Gleichzeitig handelt es sich bei der Zentrierung um ein konventionelles Vorgehen, um Multikollinearitätsproblemen zu begegnen (Jaccard et al. 1990: 31; Smith/Sasaki 1979: 39). Kam und Franzese (2007: 4) weisen allerdings darauf hin, dass diese Maßnahme nur kosmetische Effekte hat, da dabei lediglich das Prinzip des VIF-Tests mathematisch ausgehebelt, das substanzielle Problem fehlender bzw. überlappender Informationen aber nicht behoben wird (siehe auch Brambor et al. 2006: 71). Wir zentrieren die Variablen daher nicht, um Multikollinearitätsprobleme - die aufgrund der großen Anzahl der berücksichtigten Interaktionseffekte auch bei unseren Analysen bestehen - nur vermeintlich zu lösen, sondern um die Interpretation der Ergebnisse zu erleichtern.

27 Die Verteilungen der Grundhaltungen zum Atlantizismus und der Identifikation mit einer der Regierungsparteien sind jeweils linksschief. Diese Merkmale wurden um $-1 /+3 \mathrm{bzw}$. $-0,5 /+3,5$ Standardabweichungen variiert, um auch hier den substanziell bedeutsamen Ausschnitt der Verteilung zu präsentieren.

28 Die in der multivariaten Regression geschätzten Koeffizienten des Ziel-Frames und des Interaktionsterms aus Ziel-Frame und Internationalismus betragen jeweils 0,18 (siehe Tabelle 5.) Die Standardabweichung von Internationalismus beträgt 0,29 (tabellarisch nicht ausgewiesen). Der Effekt des Ziel-Frames bei Befragten, die sich auf der Internationalismus-Dimension eine Standardabweichung oberhalb des arithmetischen Mittels befinden, ergibt sich somit wie folgt: 0,18 (Koeffizient des Ziel-Frames) + 0,29 (Standardabweichung Internationalismus) $* 0,18$ (Koeffizient des Interaktionsterms) $=0,23$. 
pung des unteren Konfidenzintervalls mit der X-Achse abzulesen ist. ${ }^{29}$ Dies deutet darauf hin, dass diese Bürger - konsistent mit ihrer Grundhaltung, jegliche Involvierung Deutschlands in die internationale Politik abzulehnen - den möglichen Einsatz praktisch unabhängig von der Ziel-Dimension bewerten.

Auch ob sich Bürger politisch eher dem rechten oder dem linken Lager zuordnen, hat einen Einfluss auf die Effektstärke des Ziel-Frames. Im Vergleich zu Konservativen bewerten Linke den möglichen Einsatz der Bundeswehr in Libyen bei gleichzeitigem Verweis auf die humanitäre Zielsetzung deutlich positiver. Der Koeffizient des Ziel-Frames liegt für Befragte, die sich im Vergleich zum Durchschnitt um eine Standardabweichung weiter links auf der Links-rechts-Skala platziert haben, bei $b=0,23$. Für Befragte, die eine Standardabweichung weiter rechts als der Durchschnittsbefragte platziert sind, ergibt sich dagegen lediglich ein Koeffizient von $b=0,13$. Der Graph unten links in Abbildung 1 macht ebenfalls deutlich, dass es bei Personen, die sich weit auf der rechten Seite des politischen Spektrums einordnen, zu gar keiner Verschiebung des Zustimmungsniveaus kommt. Diese Befunde dürften auf inklusivere Solidaritätsvorstellungen zurückzuführen sein, die linke Bürger dazu veranlassen, dem Schutz libyscher Flüchtlinge eine hohe Priorität einzuräumen. Für rechte bzw. konservative Bürger wird der mögliche Einsatz dagegen nicht oder nur geringfügig unterstützungswürdiger, wenn auf die humanitäre Zielsetzung verweisen wird.

Insgesamt lässt sich somit feststellen, dass im Rahmen des framing-Experiments Bevölkerungsbewertungen eines möglichen Libyen-Einsatzes durch die Betonung der humanitären Zielsetzung in Richtung einer größeren Zustimmung verschoben werden konnten. Eine mehrheitliche Befürwortung eines solchen Bundeswehreinsatzes konnte damit allerdings nicht erreicht werden. Die Bewertungsverschiebungen waren zudem in bestimmten Bevölkerungsgruppen in stärkerem Maße zu beobachten als in anderen. Bürger, die grundsätzlich eine aktive deutsche Außenpolitik befürworteten sowie Mitglieder des linken Lagers reagierten in stärkerem Maße auf den Ziel-Frame.

\section{Diskussion und Fazit}

Ziel dieses Beitrags war es zu untersuchen, ob sich Bevölkerungsbewertungen in Deutschland zu militärischen Auslandseinsätzen mithilfe von Kommunikationsstrategien verschieben lassen. Angesichts der antimilitaristischen Grundhaltungen der Deutschen stellt sich diese Frage gerade bei Kampfeinsätzen, bei denen der militärische bzw. kriegerische Aspekt im Vordergrund steht. Am Beispiel der im Frühjahr 2011 kurzzeitig diskutierten Beteiligung der Bundeswehr an einem multilateral geführten Kampfeinsatz in Libyen lässt sich nachweisen, dass Bevölkerungsbewer-

29 Aufgrund der kleinen Zahl von Personen, die derartige Extrempositionen vertreten, wird die Schätzung der Effekte für diese Ausprägungen allerdings recht ungenau. Die Konfidenzintervalle werden daher sehr groß. 
tungen tatsächlich durch framing verschoben werden können. Das Umfrageexperiment ergab, dass es in diesem Fall bei Bürgern, die eine aktive Rolle Deutschlands in der internationalen Politik grundsätzlich befürworteten, und bei Personen, die sich dem linken Lager zuordneten, zu besonders großen Verschiebungen kam. Insgesamt blieb es allerdings stets bei einer mehrheitlichen Ablehnung, vor allem weil die kritische Grundhaltung zum Einsatz militärischer Mittel ihre Bedeutung für die Meinungsbildung auch bei dem Verweis auf die humanitäre Zielsetzung nicht verlor.

Die Befunde dieses Beitrags legen nahe, dass eine derartige Kommunikationsstrategie auch in anderen, ähnlichen Fällen wirksam sein könnte. Obwohl die Deutschen einen solchen (multilateral geführten Kampf-)Einsatz vor allem im Lichte ihrer Grundüberzeugungen zum Einsatz militärischer Mittel bewerten dürften, würde der humanitäre Aspekt von den meisten Bürgern berücksichtigt und zu einer größeren Unterstützung führen. Aufgrund der gewählten Untersuchungsmethode - ein auf einen Einzelfall bezogenes framing-Experiment innerhalb einer Online-Befragung - lassen die präsentierten Ergebnisse allerdings keinen Schluss darüber zu, ob eine derartige Kommunikationsstrategie tatsächlich eine mehrheitliche Unterstützung für einen solchen Einsatz in der deutschen Bevölkerung erzeugen könnte. Eine derartige Verallgemeinerung wäre in Anbetracht der oben diskutierten Einschränkungen der externen Validität eine zu weitreichende Schlussfolgerung. Ziel des framing-Experiments war es vielmehr, ein einzelnes Glied einer längeren Kausalkette anhand eines geeigneten Falls zu untersuchen. Erstmals konnte so der kausale Effekt der Darstellung eines Auslandseinsatzes auf den Meinungsbildungsprozess methodisch angemessen auf der Mikroebene isoliert werden. Dieses vergleichsweise reduktionistische Verfahren, das zum Kausalitätsnachweis gut geeignet ist, kann so qualitative Fallstudien und quantitative Analysen von Beobachtungsdaten, die ihre eigenen Vorzüge und Probleme aufweisen, sinnvoll ergänzen.

Die Ergebnisse deuten zudem darauf hin, dass sich Kommunikationsstrategien nicht ausschließlich auf ein Argument stützen sollten, wenn damit die Unterstützung breiter Teile der Bevölkerung gesichert werden soll. Offenbar verfangen nicht alle Darstellungen bei allen Bürgern gleich gut. Dabei scheint die Wirksamkeit einer framing-Strategie in einer gegebenen Situation nicht zufällig zu sein, sondern von bestimmten individuellen Merkmalen abzuhängen. In dem hier präsentierten Fall reagierten vor allem internationalistische, linke Bürger mit einer positiveren Bewertung. Eine andere Darstellung des Einsatzes - möglicherweise der Verweis auf die Bündnispflichten - hätte möglicherweise das konservative Lager in stärkerem Maße angesprochen und dort zu einer stärkeren Verschiebung geführt als bei den Linken. Die Wirksamkeit dieser anderen Kommunikationsstrategien zu untersuchen ist eine Frage, mit der sich die Forschung in Zukunft befassen sollte. ${ }^{30}$

30 Auch sollten die Mechanismen genauer untersucht werden, auf denen framing-Effekte beruhen. Beispielsweise könnten Angst oder andere Emotionen bei der Urteilsbildung zu außen- und sicherheitspolitischen Fragen eine Rolle spielen. Siehe zum Beispiel die Beiträge von Huddy et al. (2005) und Schoen (2006a; 2006b). 
Was hier für den möglichen Libyen-Einsatz gezeigt wurde, muss nicht für andere Militäreinsätze gleichermaßen gelten. Fälle, in denen die Friedenssicherung im Vordergrund steht und wahrscheinlich keine Kampfhandlungen stattfinden werden, könnten bei den Deutschen durchaus zunächst Vorstellungen aktivieren, die hier in dem Ziel-Frame angesprochen werden. In einem solchen Fall könnte - anders als bei dem möglichen Einsatz der Bundeswehr in Libyen - das framing des Einsatzes als gefährliche militärische Unternehmung durchaus zu einer Verschiebung der Bevölkerungsmeinungen führen. In Fällen, in denen der völkerrechtliche Status einer Intervention fraglich ist und im Zentrum der Debatte steht, könnten zudem die beiden hier berücksichtigten Dimensionen in den Hintergrund treten. Ebenso könnte eine stärker entlang von Parteilinien geführte Debatte die Wirksamkeit von framing-Strategien beeinflussen.

Militärische Auslandseinsätze werden aller Voraussicht nach auch in Zukunft ein Thema sein, mit dem sich politische Entscheidungsträger und die Bevölkerung in Deutschland auseinandersetzen müssen. Das wechselseitige Verhältnis zwischen beiden zu untersuchen verspricht dabei wichtige Erkenntnisse über die Faktoren, die die deutsche Außenpolitik beeinflussen, nicht zuletzt aber über die Rolle der Regierung in diesem Prozess. Dieses Potential sollte Anreiz genug sein, auch in Zukunft Diskurse über diese Themen aufmerksam zu verfolgen und sorgfältig zu untersuchen. Denn wie eng die Grenzen sind, die die öffentliche Meinung der Handlungsfreiheit politischer Entscheidungsträger setzt, scheint auch davon abzuhängen, wie Entscheidungen im öffentlichen Diskurs dargestellt werden. Die Möglichkeiten und Grenzen dieser Kommunikationsstrategien auszuloten, lässt Rückschlüsse auf den Grad der demokratischen Kontrolle zu. Ob deutsche Politiker den Spielraum, den ihnen Kommunikationsstrategien offenbar grundsätzlich verschaffen können, bei der nächsten Diskussion um einen militärischen Auslandseinsatz nutzen, wird die Zukunft zeigen. 


\section{Anhang: Operationalisierungen ${ }^{31}$}

\section{Framing-Experiment}

3-er Split: Die folgenden Fragen werden jeweils an ein Drittel der Befragten gestellt.

»In Europa und Deutschland wird diskutiert, in Libyen militärische Einsätze durchzuführen. Befürworten Sie die Teilnahme deutscher Soldaten an solchen Einsätzen in Libyen oder lehnen Sie diese ab?«

\begin{tabular}{|c|c|c|c|c|c|c|c|c|c|c|c|}
\hline & $\begin{array}{l}\text { Lehne Ein- } \\
\text { satz voll } \\
\text { und ganz ab } \\
-5\end{array}$ & -4 & -3 & -2 & -1 & 0 & 1 & 2 & 3 & 4 & $\begin{array}{l}\text { Stimme Ein- } \\
\text { satz voll und } \\
\text { ganz zu } \\
5\end{array}$ \\
\hline $\mathrm{N}(341)$ & 171 & 25 & 27 & 11 & 13 & 40 & 9 & 16 & 13 & 2 & 14 \\
\hline$\%$ & 50,2 & 7,3 & 7,9 & 3,2 & 3,8 & 11,7 & 2,6 & 4,7 & 3,8 & 0,6 & 4,11 \\
\hline
\end{tabular}

»In Europa und Deutschland wird diskutiert, in Libyen militärische Einsätze mit Bodentruppen durchzuführen. Dabei bestünde natürlich auch die Gefahr, dass die Soldaten in Kampfhandlungen verwickelt werden. Befürworten Sie die Teilnahme deutscher Soldaten an solchen Einsätzen in Libyen oder lehnen Sie diese ab?«

\begin{tabular}{cccccccccccc}
\hline & $\begin{array}{c}\text { Lehne Ein- } \\
\text { satz voll } \\
\text { und ganz ab }\end{array}$ & & & & & & & & & & $\begin{array}{c}\text { Stimme Ein- } \\
\text { satz voll und } \\
\text { ganz zu }\end{array}$ \\
& -5 & -4 & -3 & -2 & -1 & 0 & 1 & 2 & 3 & 4 & 5 \\
\hline $\mathrm{N}(347)$ & 156 & 26 & 26 & 18 & 11 & 37 & 20 & 22 & 18 & 4 & 9 \\
$\%$ & 45,0 & 7,5 & 7,5 & 5,2 & 3,2 & 10,7 & 5,8 & 6,3 & 5,2 & 1,2 & 2,6 \\
\hline
\end{tabular}

»In Europa und Deutschland wird diskutiert, in Libyen humanitäre Einsätze mit militärischen Mitteln zu schützen. Dies soll es beispielsweise ermöglichen, Flüchtlinge in Sicherheit zu bringen. Befürworten Sie die Teilnahme deutscher Soldaten an solchen Einsätzen in Libyen oder lehnen Sie diese ab?«

\begin{tabular}{|c|c|c|c|c|c|c|c|c|c|c|c|}
\hline & $\begin{array}{c}\text { Lehne Ein- } \\
\text { satz voll } \\
\text { und ganz ab } \\
-5\end{array}$ & -4 & -3 & -2 & -1 & 0 & 1 & 2 & 3 & 4 & $\begin{array}{l}\text { Stimme Ein- } \\
\text { satz voll und } \\
\text { ganz zu } \\
5\end{array}$ \\
\hline $\mathrm{N}(345)$ & 90 & 16 & 21 & 15 & 16 & 48 & 18 & 43 & 41 & 11 & 26 \\
\hline$\%$ & 26,1 & 4,6 & 6,1 & 4,4 & 4,6 & 13,9 & 5,2 & 12,5 & 11,9 & 3,2 & 7,5 \\
\hline
\end{tabular}

31 Es wird der Wertebereich der Originaldaten angegeben. Bei der Angabe der Randverteilungen ergibt die Summe der Zeilenprozente aufgrund von Rundungsfehlern nicht immer genau $100 \%$. 


\section{Aufsätze}

\section{Militarismus}

»Der Einsatz militärischer Gewalt ist niemals gerechtfertigt.»

\begin{tabular}{cccccc}
\hline & $\begin{array}{c}\text { Stimme } \\
\text { überhaupt } \\
\text { nicht zu } \\
1\end{array}$ & 2 & 3 & 4 & $\begin{array}{c}\text { Stimme } \\
\text { voll und } \\
\text { ganz zu }\end{array}$ \\
\hline $\mathrm{N}(1035)$ & 142 & 169 & 295 & 200 & 5 \\
$\%$ & 13,7 & 16,3 & 28,5 & 19,3 & 229 \\
\hline
\end{tabular}

»Krieg ist manchmal notwendig, um die Interessen eines Landes zu schützen.»

\begin{tabular}{cccccc}
\hline & $\begin{array}{c}\text { Stimme } \\
\text { überhaupt } \\
\text { nicht zu } \\
1\end{array}$ & 2 & 3 & 4 & $\begin{array}{c}\text { Stimme } \\
\text { voll und } \\
\text { ganz zu } \\
5\end{array}$ \\
\hline $\mathrm{N}(1036)$ & 344 & 195 & 253 & 151 & 93 \\
$\%$ & 33,2 & 18,8 & 24,4 & 14,6 & 9,0 \\
\hline
\end{tabular}

Item 1 für Indexbildung recodiert, Reliabilitätsmaß: $\alpha=0,67$

\section{Internationalismus}

»Deutschland sollte eine aktivere Rolle in der Weltpolitik spielen.«

\begin{tabular}{cccccc}
\hline & $\begin{array}{c}\text { Stimme } \\
\text { überhaupt } \\
\text { nicht zu } \\
1\end{array}$ & 2 & 3 & 4 & $\begin{array}{c}\text { Stimme } \\
\text { voll und } \\
\text { ganz zu } \\
5\end{array}$ \\
\hline $\mathrm{N}(1019)$ & 114 & 137 & 370 & 268 & 130 \\
$\%$ & 11,2 & 13,4 & 36,1 & 26,3 & 12,8 \\
\hline
\end{tabular}




\section{Multilateralismus}

»Bei einer internationalen Krise sollten sich Deutschland und seine Verbündeten auf eine gemeinsame Haltung einigen."

\begin{tabular}{cccccc}
\hline & $\begin{array}{c}\text { Stimme } \\
\text { überhaupt } \\
\text { nicht zu } \\
1\end{array}$ & 2 & 3 & 4 & $\begin{array}{c}\text { Stimme } \\
\text { voll und } \\
\text { ganz zu } \\
5\end{array}$ \\
\hline $\mathrm{N}(1032)$ & 50 & 53 & 238 & 298 & 393 \\
$\%$ & 4,8 & 5,1 & 23,0 & 28,9 & 38,1 \\
\hline
\end{tabular}

»Für seine Sicherheit sollte Deutschland vor allem alleine sorgen.«

\begin{tabular}{cccccc}
\hline & $\begin{array}{c}\text { Stimme } \\
\text { überhaupt } \\
\text { nicht zu } \\
1\end{array}$ & 2 & 3 & 4 & $\begin{array}{c}\text { Stimme } \\
\text { voll und } \\
\text { ganz zu } \\
5\end{array}$ \\
\hline $\mathrm{N}(1039)$ & 110 & 182 & 330 & 215 & 202 \\
$\%$ & 10,6 & 17,5 & 31,8 & 20,7 & 19,4 \\
\hline
\end{tabular}

Item 2 für Indexbildung recodiert, Reliabilitätsmaß: $\alpha=0,27$ 


\section{Aufsätze}

\section{Atlantizismus}

»In außenpolitischen Fragen sollte Deutschland in Übereinstimmung mit den USA handeln.«

\begin{tabular}{cccccc}
\hline & $\begin{array}{c}\text { Stimme } \\
\text { überhaupt } \\
\text { nicht zu } \\
1\end{array}$ & 2 & 3 & 4 & $\begin{array}{c}\text { Stimme } \\
\text { voll und } \\
\text { ganz zu }\end{array}$ \\
\hline $\mathrm{N}(1028)$ & 312 & 247 & 296 & 101 & 5 \\
$\%$ & 30,4 & 24,0 & 28,8 & 9,8 & 72 \\
\hline
\end{tabular}

»Deutschland sollte seine Interessen gegenüber den USA selbstbewusster vertreten.«

\begin{tabular}{cccccc}
\hline & $\begin{array}{c}\text { Stimme } \\
\text { überhaupt } \\
\text { nicht zu } \\
1\end{array}$ & 2 & 3 & 4 & $\begin{array}{c}\text { Stimme } \\
\text { voll und } \\
\text { ganz zu } \\
5\end{array}$ \\
\hline $\mathrm{N}(1024)$ & 14 & 36 & 231 & 329 & 414 \\
\hline & 1,4 & 3,5 & 22,6 & 32,1 & 40,3 \\
\hline
\end{tabular}

Item 2 für Indexbildung recodiert, Reliabilitätsmaß: $\alpha=0,33$

\section{Links-rechts-Orientierung}

»In der Politik reden die Leute häufig von links und rechts. Wenn Sie eine Skala von 1 bis 11 benutzen, wo würden Sie sich selbst einordnen?«

\begin{tabular}{cccccccccccc}
\hline & Links & & & & & & & \multicolumn{2}{c}{ Rechts } \\
& 1 & 2 & 3 & 4 & 5 & 6 & 7 & 8 & 9 & 10 & 11 \\
\hline $\mathrm{N}(1073)$ & 54 & 44 & 115 & 122 & 128 & 300 & 104 & 90 & 56 & 20 & 40 \\
$\%$ & 5,0 & 4,1 & 10,7 & 11,4 & 11,9 & 28,0 & 9,7 & 8,4 & 5,2 & 1,9 & 3,7 \\
\hline
\end{tabular}

\section{Regierungs-PID}

»Und jetzt noch einmal kurz zu den politischen Parteien. In Deutschland neigen viele Leute längere Zeit einer bestimmten politischen Partei zu, obwohl sie auch ab und zu eine andere Partei wählen. Wie ist das bei Ihnen: Neigen Sie - ganz allgemein - einer bestimmten Partei zu? Und wenn ja, welcher?«(Wertebereich: 0,1)

\begin{tabular}{ccc}
\hline & Keine & Regierungs-PID \\
& Regierungs-PID & 265 \\
\hline $\mathrm{N}(1216)$ & 951 & 21,79 \\
$\%$ & 78,2 & \\
\hline
\end{tabular}




\section{Interesse}

»Man kann sich ja nicht in allen Politikbereichen gleich gut auskennen. Wie stark interessieren Sie sich für Verteidigungsfragen und Außenpolitik?« (Wertebereich: 1 bis 5)

\begin{tabular}{cccccc}
\hline & $\begin{array}{c}\text { Überhaupt } \\
\text { nicht interessiert }\end{array}$ & & & \multicolumn{2}{c}{ Sehr stark interessiert } \\
& 1 & 2 & 3 & 4 & 5 \\
\hline $\mathrm{N}(1136)$ & 117 & 221 & 488 & 229 & 81 \\
$\%$ & 10,3 & 19,5 & 43,0 & 20,2 & 7,1 \\
\hline
\end{tabular}

$\begin{array}{ll}\text { Alter } & \text { in Jahren } \\ \text { Bildung } & 0: \text { andere Befrage } \\ & 1: \text { Abiturient } \\ \text { Geschlecht } & 0: \text { männlich } \\ & 1: \text { weiblich } \\ \text { Herkunft } & 0: \text { andere Herkunft } \\ & 1: \text { neue Bundesländer }\end{array}$

\section{Literatur}

Aldrich, John H./Gelpi, Christopher/Feaver, Peter/Reifler, Jason/Sharp, Kristin Thompson 2006: Foreign Policy and the Electoral Connection, in: Annual Review of Political Science 9, 477-502.

Aldrich, John H./Sullivan, John L./Borgida, Eugene 1989: Foreign Affairs and Issue Voting: Do Presidential Candidates »Waltz Before a Blind Audience?«, in: American Political Science Review 83: 1, 123-141.

Arzheimer, Kai 2009: Ideologien, in: Kaina, Viktoria/Römmele, Andrea (Hrsg.): Politische Soziologie. Ein Studienbuch, Wiesbaden, 83-108.

Asmus, Ronald D./Everts, Philip P./Isernia, Pierangelo 2005: The Transatlantic Gap in Public Opinion, in: Jäger, Thomas/Höse, Alexander/Oppermann, Kai (Hrsg.): Transatlantische Beziehungen. Sicherheit - Wirtschaft - Öffentlichkeit, Wiesbaden, 397-424.

Baum, Matthew A./Groeling, Tim J. 2009: Shot by the Messenger: Partisan Cues and Public Opinion Regarding National Security and War, in: Political Behavior 31: 2, 157-186.

Baumann, Rainer/Hellmann, Gunther 2001: Germany and the Use of Force: »Total War«, the »Culture of Restraint« and the Quest for Normality, in: German Politics 10: 1, 61-82.

Berger, Thomas U. 1998: Cultures of Antimilitarism: National Security in Germany and Japan, Chicago, IL.

Biehl, Heiko/Fiebig, Rüdiger/Giegerich, Bastian/Jacobs, Jörg/Jonas, Alexandra (Hrsg.) 2011: Strategische Kulturen in Europa. Die Bürger Europas und ihre Streitkräfte. Ergebnisse der Bevölkerungsbefragungen in acht europäischen Ländern 2010 des Sozialwissenschaftlichen Instituts der Bundeswehr (Forschungsbericht 96), in: https:/dgap.org/de/ article/getFullPDF/20552; 23.4.2013.

Brambor, Thomas/Clark, William Roberts/Golder, Matt 2006: Understanding Interaction Models: Improving Empirical Analyses, in: Political Analysis, 14: 1, 63-82.

Brooks, Clem/Dodson, Kyle/Hotchkiss, Nikole 2010: National Security Issues and US Presidential Elections: 1992-2008, in: Social Science Research 39: 4, 518-526.

Campbell, Angus/Converse, Philip E./Miller, Warren E./Stokes, Donald E. 1960: The American Voter, Chicago, Il. 
Caprara, Gian Vittorio/Schwartz, Shalom/Capanna, Cristina/Vecchione, Michele/Barbaranelli, Claudio 2006: Personality and Politics: Values, Traits, and Political Choice, in: Political Psychology 27: 1, 1-28.

Chittick, William O./Billingsley, Keith R./Travis, Rick 1995: A Three-Dimensional Model of American Foreign Policy Beliefs, in: International Studies Quarterly 39: 3, 313-331.

Chong, Dennis/Druckman, James N. 2007a: Framing Theory, in: Annual Review of Political Science 10: 1, 103-126.

Chong, Dennis/Druckman, James N. 2007b: A Theory of Framing and Opinion Formation in Competitive Elite Environments, in: Journal of Communication 57: 1, 99-118.

Chong, Dennis/Druckman, James N. 2010: Dynamic Public Opinion: Communication Effects over Time, in: American Political Science Review 104: 4, 663-680.

Druckman, James N. 2001a: The Implications of Framing Effects for Citizen Competence, in: Political Behavior 23: 3, 225-256.

Druckman, James N. 2001b: On the Limits of Framing Effects: Who Can Frame?, in: Journal of Politics 63: 4, 1041-1066.

Druckman, James N. 2004: Political Preference Formation: Competition, Deliberation, and the (Ir)relevance of Framing Effects, in: American Political Science Review 98: 4, 671-686.

Druckman, James N./Green, Donald P./Kuklinski, James H./Lupia, Arthur (Hrsg.) 2011: Cambridge Handbook of Experimental Political Science, New York, NY.

Druckman, James N./Fein, Jordan/Leeper, Thomas J. 2012: A Source of Bias in Public Opinion Stability, in: American Political Science Review 106: 2, 430-454.

Druckman, James N./Leeper, Thomas J. 2012: Learning More from Political Communication Experiments: Pretreatment and Its Effects, in: American Journal of Political Science 56: 4, 875-896.

Druckman, James N./Nelson, Kjersten R. 2003: Framing and Deliberation: How Citizens' Conversations Limit Elite Influence, in: American Journal of Political Science 47: 4, 729-745.

Duffield, John S. 1998: World Power Forsaken: Political Culture, International Institutions, and German Security Policy after Unification, Stanford, CA.

Eagley, Alice H./Chaiken, Shelly 1993: The Psychology of Attitudes, Fort Worth, TX.

Faas, Thorsten/Schoen, Harald 2006: Putting a Questionnaire on the Web Is not Enough: A Comparison of Online and Offline Surveys Conducted in the Context of the German Federal Election 2002, in: Journal of Official Statistics, 22: 2, 177-190.

Fazio, Russell H. 2007: Attitudes as Object-Evaluation Associations of Varying Strength, in: Social Cognition 25: 5, 603-637.

Feldman, Stanley 1988: Structure and Consistency in Public Opinion: The Role of Core Beliefs and Values, in: American Journal of Political Science 32: 2, 416-440.

Fiebig, Rüdiger 2008: Sicherheits- und verteidigungspolitische Einstellungen im Vergleich. Deutschland, Frankreich, Großritannien, USA, in: Bulmahn, Thomas/Fiebig, Rüdiger/ Sender, Wolfgang (Hrsg.): Sicherheits- und verteidigungspolitisches Meinungsklima in der Bundesrepublik Deutschland. Ergebnisse der Bevölkerungsbefragung 2006 des Sozialwissenschaftlichen Instituts der Bundeswehr (Forschungsbericht 84), 81-88, in: http:// www.mgfa.de/html/einsatzunterstuetzung/downloads/forschungsbericht 84 mitfragebogen.pdf?PHPSESSID=931748af0e86616800373655acaf2902; 9.3.2013.

Fishbein, Martin/Ajzen, Icek 1975: Belief, Attitude, Intention and Behavior: An Introduction to Theory and Research, Reading, MA.

Gareis, Sven Bernhard 2011: Militärische Auslandseinsätze und die Transformation der Bundeswehr, in: Jäger, Thomas/Höse, Alexander/Oppermann, Kai (Hrsg.): Deutsche Außenpolitik, 2., aktualisierte und erweiterte Aufl., Wiesbaden, 148-170.

Gerber, Alan S./Gimpel, James G./Green, Donald P./Shaw, Daron R. 2011: How Large and Long-lasting Are the Persuasive Effects of Televised Campaign Ads? Results from a Randomized Field Experiment, in: American Political Science Review 105: 1, 135-150.

Göler, Daniel 2010: Die strategische Kultur der Bundesrepublik - Eine Bestandsaufnahme normativer Vorstellungen über den Einsatz militärischer Mittel, in: Dörfler-Dierken, An- 
gelika/Portugall, Gerd (Hrsg.): Friedensethik und Sicherheitspolitik, Wiesbaden, 185-199.

Green, Donald P./Ha, Shang E./Bullock, John G. 2010: Enough Already about »Black Box" Experiments: Studying Mediation Is More Difficult than Most Scholars Suppose, in: The Annals of the American Academy of Political and Social Science 628: 1, 200-208.

Green, Donald P./Palmquist, Bradley/Schickler, Eric 2002: Partisan Hearts and Minds: Political Parties and the Social Identities of Voters, New Haven, CT.

Greene, Steven 1999: Understanding Party Identification, in: Political Psychology 20: 2, 393-403.

Hellmann, Gunther 2011: Berlins Große Politik im Fall Libyen, in: Welttrends, 80: September/Oktober, 19-22.

Hermann, Margaret G./Ozkececi-Taner, Binnur 2011: The Experiment and Foreign Policy Decision Making, in: Druckman, Daniel N./Green, Donald P./Kuklinski, James H./ Lupia, Arthur (Hrsg.): Cambridge Handbook of Experimental Political Science, New York, NY, 430-442.

Ho, Daniel E./Imai, Kosuke/King, Gary/Stuart, Elizabeth A. 2007: Matching as Nonparametric Preprocessing for Reducing Model Dependence in Parametric Causal Inference, in: Political Analysis 15: 3, 199-236.

Holsti, Ole R. 2004: Public Opinion and American Foreign Policy, 2. überarb. Aufl., Ann Arbor, MI.

Huddy, Leonie/Feldman, Stanley/Taber, Charles/Lahav, Gallya 2005: Threat, Anxiety, and Support of Anti-Terrorism Policies, in: American Journal of Political Science, 49: 3, 610-625.

Hurwitz, Jon/Peffley, Mark A. 1987: How Are Foreign Policy Attitudes Structured? A Hierarchical Model, in: American Political Science Review 81: 4, 1099-1120.

Imai, Kosuke/Keele, Luke/Tingley, Dustin/Yamamoto, Teppei 2011: Unpacking the Black Box of Causality: Learning about Causal Mechanisms from Experimental and Observational Studies, in: American Political Science Review 105: 4, 765-789.

Imai, Kosuke/King, Gary/Stuart, Elizabeth A. 2008: Misunderstandings between Experimentalists and Observationalists about Causal Inference, in: Journal of the Royal Statistical Society: Series A (Statistics in Society) 171: 2, 481-502.

Isernia, Pierangelo/Everts, Philip 2004: Partners Apart? The Foreign Policy Attitudes of the American and European Publics, in: Japanese Journal of Political Science 5: 2, 229-258.

Jaccard, James/Turrisi, Robert/Wan, Choi K. 1990: Interaction Effects in Multiple Regression, Newbury Park, CA.

Jacobs, Lawrence R./Shapiro, Robert Y. 1999: Lyndon Johnson, Vietnam, and Public Opinion: Rethinking Realist Theory of Leadership, in: Presidential Studies Quarterly 29: 3, 592-616.

Johnston, Alastair Iain 1995: Thinking about Strategic Culture, in: International Security 19: 4, 32-64.

Juhász, Zoltán 1996: Außenpolitische Denkbilder. Eine empirische Untersuchung außenpolitischer Einstellungen in der deutschen Bevölkerung nach der Wiedervereinigung, Hamburg.

Juhász, Zoltán 2001: German Public Opinion and the Use of Force in the Early 1990s, in: Everts, Philip/Isernia, Pierangelo (Hrsg.): Public Opinion and the International Use of Force, London, 57-85.

Kam, Cindy D./Franzese, Robert J. 2007: Modeling and Interpreting Interactive Hypotheses in Regression Analysis, Ann Arbor, MI.

Kuklinski, James H./Hurley, Norman L. 1994: On Hearing and Interpreting Political Messages: A Cautionary Tale of Citizen Cue-Taking, in: The Journal of Politics 56: 3, 729-751.

Kühnel, Steffen M./Krebs, Dagmar 2001: Statistik für Sozialwissenschaftler. Grundlagen, Methoden, Anwendungen, Hamburg. 
Lupia, Arthur 1992: Busy Voters, Agenda Control, and the Power of Information, in: American Political Science Review 86: 2, 390-403.

Mair, Peter 2007: Left-Right Orientations, in: Dalton, Russell J./Klingemann, Hans-Dieter (Hrsg.): The Oxford Handbook of Political Behavior, Oxford, 206-222.

Maull, Hanns W. 2000: Germany and the Use of Force: Still a Civilian Power?, in: Survival, 42: $2,56-80$.

Maull, Hanns W. 2011: Deutsche Außenpolitik. Orientierungslos, in: Zeitschrift für Politikwissenschaft 21: 1, 93-117.

McDermott, Rose 2011a: Internal and External Validity, in: Druckman, Daniel N./Green, Donald P./Kuklinski, James H./Lupia, Arthur (Hrsg.): Cambridge Handbook of Experimental Political Science, New York, NY, 27-40.

McDermott, Rose 2011b: New Directions for Experimental Work in International Relations, in: International Studies Quarterly 55: 2, 503-520.

Meiers, Franz-Josef 2010: Von der Scheckbuchdiplomatie zur Verteidigung am Hindukusch. Die Rolle der Bundeswehr bei multinationalen Auslandseinsätzen 1990-2009, in: Zeitschrift für Außen- und Sicherheitspolitik, 3: 2, 201-222.

Merkel, Angela 2011: Abgabe einer Regierungserklärung durch die Bundeskanzlerin zum Europäischen Rat am 24./25. März 2011 in Brüssel (Deutscher Bundestag - 17. Wahlperiode - 99. Sitzung, 24.3.2011, Plenarprotokoll 17/99), in: http://dipbt.bundestag.de/ $\operatorname{dip} 21 / \mathrm{btp} / 17 / 17099 . p d f: 7.1 .2013$.

Miller, Joanne M./Krosnick, Jon A. 2000: News Media Impact on the Ingredients of Presidential Evaluations: Politically Knowledgeable Citizens Are Guided by a Trusted Source, in: American Journal of Political Science 44: 2, 301-315.

Mintz, Alex/Yang, Yi/McDermott, Rose 2011: Experimental Approaches to International Relations, in: International Studies Quarterly 55: 2, 493-501.

Moravcsik, Andrew 1997: Taking Preferences Seriously: A Liberal Theory of International Politics, in: International Organization 51: 4, 513-553.

Müller, Harald 2011: Ein Desaster. Deutschland und der Fall Libyen. Wie sich Deutschland moralisch und außenpolitisch in die Isolation manövrierte, HSFK Standpunkte 2011: 2.

Müller, Harald/Fey, Sabine/Mannitz, Sabine/Schörnig, Niklas 2010: Demokratie, Streitkräfte und militärische Einsätze. Der »zweite Gesellschaftsvertrag« steht auf dem Spiel, HSFK-Report 2010: 10.

Nelson, Thomas E./Kinder, Donald R. 1996: Issue Frames and Group-Centrism in American Public Opinion, in: The Journal of Politics 58: 4, 1055-1078.

Nelson, Thomas E./Oxley, Zoe M./Clawson, Rasalee A. 1997: Towards a Psychology of Framing Effects, in: Political Behavior 19: 3, 221-246.

Nincic, Miroslav/Ramos, Jennifer M. 2010: Ideological Structures and Foreign Policy Preferences, in: Journal of Political Ideologies 15: 2, 119-141.

Norpoth, Helmut/Sidman, Andrew H. 2007: Mission Accomplished: The Wartime Election of 2004, in: Political Behavior 29: 2, 175-195.

Page, Benjamin I./Shapiro, Robert Y. 1983: Effects of Public Opinion on Policy, in: American Political Science Review 77: 1, 175-190.

Philippi, Nina 1997: Bundeswehr-Auslandseinsätze als außen- und sicherheitspolitisches Problem des geeinten Deutschland, Frankfurt a. M.

Putnam, Robert D. 1988: Diplomacy and Domestic Politics: The Logic of Two-Level Games, in: International Organization 42: 3, 427-460.

Pötzschke, Jana/Rattinger, Hans/Schoen, Harald 2013: Persönlichkeit, Wertorientierungen und Einstellungen zu Außen- und Sicherheitspolitik in den Vereinigten Staaten, in: Politische Psychologie, i.E.

Rathbun, Brian C. 2007: Hierarchy and Community at Home and Abroad: Evidence of a Common Structure of Domestic and Foreign Policy Beliefs in American Elites, in: Journal of Conflict Resolution 51: 3, 379-407. 
Rattinger, Hans 1996: Einstellungen zur europäischen Integration in der Bundesrepublik: Ein Kausalmodell, in: Zeitschrift für Internationale Beziehungen 3: 1, 45-78.

Rattinger, Hans/Holst, Christian 1998: Strukturen und Determinanten außen- und sicherheitspolitischer Einstellungen in der Bundesrepublik, in: http:/www.ssoar.info/ssoar/handle/ document/5492; 7.1.2013.

Rattinger, Hans/Schmitt-Beck, Rüdiger/Roßteutscher, Sigrid/Weßels, Bernhard 2011: GLES 2009 Langfrist-Online-Tracking, T14 (ZA5347, Version 1.0.0). Studienbeschreibung (GESIS-Study Material 2011|91), in: http://infol.gesis.org/dbksearch/download.asp? $\mathrm{id}=20519 ; 9.3 .2013$.

Risse, Thomas 2004: Kontinuität durch Wandel: Eine »neue« deutsche Außenpolitik?, in: Aus Politik und Zeitgeschichte, B 11, 24-31.

Sanders, David/Clarke, Harold D./Stewart, Marianne C./Whiteley, Paul 2007: Does Mode Matter for Modeling Political Choice? Evidence from the 2005 British Election Study, in: Political Analysis 15: 3, 257-285.

Schoen, Harald 2006a: Beeinflusst Angst politische Einstellungen? Eine Analyse der öffentlichen Meinung zum Golfkrieg 1991, in: Politische Vierteljahresschrift 47: 3, 441-464.

Schoen, Harald 2006b: Angst und Einstellungen zum Kosovokrieg. Eine Analyse der öffentlichen Meinung in West- und Ostdeutschland, in: Zeitschrift für Internationale Beziehungen 13: 2, 177-199.

Schoen, Harald 2010: Ein Bericht von der Heimatfront. Bürger, Politiker und der Afghanistaneinsatz der Bundeswehr, in: Politische Vierteljahresschrift 51: 3, 395-408.

Schoen, Harald 2011: Two Indicators, One Conclusion: On the Public Salience of Foreign Affairs in Pre- and Post-Unification Germany, in: Oppermann, Kai/Viehrig, Henrike (Hrsg.): Issue Salience in International Politics, New York, NY.

Schörnig, Niklas 2009: In der Opferfalle. Die Bundesregierung und die zunehmenden Gefallenen der Bundeswehr, HSFK Standpunkte 2009: 2.

Schwartz, Shalom H./Caprara, Gian Vittorio/Vecchione, Michele 2010: Basic Personal Values, Core Political Values, and Voting: A Longitudinal Analysis, in: Political Psychology $31: 3,421-452$.

Smith, Kent W./Sasaki, Maurício S. 1979: Decreasing Multicollinearity, in: Sociological Methods \& Research 8: 1, 35-56.

Sniderman, Paul M./Theriault, Sean M. 2004: The Structure of Political Argument and the Logic of Issue Framing, in: Saris, Willem E./Sniderman, Paul M. (Hrsg.): Studies in Public Opinion: Attitudes, Nonattitudes, Measurement Error, and Change, Princeton, NJ, 133-165.

Snyder, Jack L. 1977: The Soviet Strategic Culture: Implications for Limited Nuclear Operations, Santa Monica, CA.

Sobel, Richard 2001: The Impact of Public Opinion on U.S. Foreign Policy since Vietnam: Constraining the Colossus, New York, NY.

Tajfel, Henri/Turner, John C. 1979: An Integrative Theory of Intergroup Conflict, in: Austin, William C./Stephen, Worchel (Hrsg.): The Social Psychology of Intergroup Relations, Monterey, CA, 33-47.

Thórisdóttir, Hulda/Jost, John T./Liviatan, Ido/Shrout, Patrick E. 2007: Psychological Needs and Values Underlying Left-Right Political Orientation: Cross-National Evidence from Eastern and Western Europe, in: Public Opinion Quarterly 71: 2, 175-203.

Turner, John C. 1982: Towards a Cognitive Redefinition of the Social Group, in: Tajfel, Henri (Hrsg.): Social Identity and Intergroup Relations, Cambridge, 15-40.

Weisberg, Herbert F./Christenson, Dino P. 2007: Changing Horses in Wartime? The 2004 Presidential Election, in: Political Behavior 29: 2, 279-304.

Westerwelle, Guido 2011: Abgabe einer Regierungserklärung durch den Bundesminister des Auswärtigen: Umbruch in der Arabischen Welt am 16. März 2011 (Deutscher Bundestag - 17. Wahlperiode - 95. Sitzung, 16.3.2011, Plenarprotokoll 17/95), in: http:// dipbt.bundestag.de/dip21/btp/17/17095.pdf: 29.3.2013. 
Wittig, Peter 2011: Statement on the Situation in Libya (Security Council Meeting on the Situation in Lybia, 6498th Security Council Meeting, 17.3.2011, S/PV.6498), in: http:// responsibilitytoprotect.org/Security\%20-Council\%20meeting\%20on\%20the $\% 20$ situation\%20in\%20Lybia\%2017\%20March\%202011.pdf: 7.1.2013.

Wittlinger, Ruth 2010: German National Identity in the Twenty-First Century: A Different Republic After All?, Basingstoke.

Zaller, John R. 1992: The Nature and Origins of Mass Opinion, New York, NY. 